\title{
BOUNDARY LAYER TAILS IN PERIODIC HOMOGENIZATION
}

\author{
GréGoire Allaire ${ }^{1}$ ANd Micol Amar ${ }^{2}$
}

\begin{abstract}
This paper focus on the properties of boundary layers in periodic homogenization in rectangular domains which are either fixed or have an oscillating boundary. Such boundary layers are highly oscillating near the boundary and decay exponentially fast in the interior to a non-zero limit that we call boundary layer tail. The influence of these boundary layer tails on interior error estimates is emphasized. They mainly have two effects (at first order with respect to the period $\varepsilon$ ): first, they add a dispersive term to the homogenized equation, and second, they yield an effective Fourier boundary condition.
\end{abstract}

Résumé. Cet article est consacré à l'étude des couches limites en homogénéisation périodique dans des domaines rectangulaires qui, soit sont fixes, soit possèdent une frontière oscillante. Ces couches limites sont très oscillantes près du bord et décroissent exponentiellement vite à l'intérieur vers une limite non nulle que nous appelons queue de couche limite. L'influence de ces queues de couches limites pour l'obtention d'estimations d'erreur intérieures est mise à jour. Elles ont pour effets principaux (au premier ordre en $\varepsilon$, la période) : premièrement, d'ajouter un terme dispersif dans l'équation homogénéisée, deuxièmement, de conduire à une condition aux limites effective de type Fourier.

AMS Subject Classification. 35B27.

Received August 31, 1998. Revised January 21, 1999.

\section{INTRODUCTION}

This paper is concerned with the study of boundary layers in periodic homogenization. Although periodic homogenization has been intensively studied ( $c f$. the books $[11,13,27])$ and boundary layers have been the topic of many works (e.g. $[10,14-16,18,21,22,26])$, there is still a lot to be understood concerning the effects of boundary layers on correctors or error estimates. Our goal is to investigate the influence at first order of these boundary layers in periodic homogenization problems posed in fixed bounded domains with, or without, an oscillating boundary.

Our motivation comes from several type of applications. Let us describe briefly some of them (our list of references is far from being complete in this wide area). First, we consider the homogenization of eigenvalue problems (with application to wave propagation in periodic media, vibration of coupled fluid-structure systems, controlability). Recent works have shown that either correctors for the low-frequency eigenvalues [25, 29], or part of the high-frequency limit spectrum $[6,7]$ are determined by boundary layers. Second, we have in mind

\footnotetext{
Keywords and phrases: Boundary layers, periodic functions, asymptotic expansion, homogenization.

${ }^{1}$ Laboratoire d'Analyse Numérique, Université Pierre et Marie Curie, Paris VI, Tour 55-65, 4 place Jussieu, 70005 Paris, France; e-mail: allaire@ann.jussieu.fr

2 Dipartimento Me.Mo.Mat, Università "La Sapienza" - Roma, Via A. Scarpa 16, 00161 Roma, Italy;

e-mail: amar@dmmm.uniroma1.it
}

(c) EDP Sciences, SMAI 1999 
the homogenization of periodically oscillating boundaries. Such problems arise in the context of fluid flows over a rough surface $[3,4]$, of reinforcement by a thin layer [17], or of electromagnetic scattering by an obstacle with a periodic coating $[1,2,20]$. In such cases the boundary layers are replaced by an effective boundary condition for the homogenized equation. Third, our results could be applied to the homogenization of neutronic diffusion or transport equation. Indeed, as reported in e.g. $[5,12,14]$, boundary layers are often more important for improving the rate of convergence than the usual periodic correctors. Fourth, studying boundary layers is also the key for determining interface transmission conditions in various mechanical problems $[10,15,22]$.

In truth we are not interested in computing exactly the full set of boundary layers (neither are we interested in obtaining a complete asymptotic expansion, valid at any order). Rather, we seek the non-oscillating tails of such boundary layers away from the boundary, and we determine if their knowledge improves, or not, the convergence rate of the homogenization process. It turns out that these boundary layers tails can be incorporated into the homogenized equation by adding dispersive terms and a Fourier boundary condition (this was already recognized by some experts, see e.g. [28], but not well described in the literature). We therefore derived optimal interior estimates using these simple boundary layer tails.

Another feature of our work is that we focus on error estimates in the $L^{2}$-norm rather than in the $H^{1}$-norm as usual. The reason for this is that, in many applications (as neutronics, for instance $[5,12]$ ), it is preferable to have a good approximation of the unknown itself rather than of its gradient. Boundary layers are often negligible for interior error estimates (i.e. away from the boundary) in the $H^{1}$-norm at first order, but not in the $L^{2}$-norm at second-order (recall that the $L^{2}$ error estimate at first-order is trivial). Part of the novelty of our work comes from this focus on higher order interior error estimates in the $L^{2}$-norm.

One drawback of this paper (like all other previous works on boundary layers in periodic homogenization) is that our analysis of these phenomena works only for rectangular domains with flat boundaries aligned with the periodicity cell.

In the case of a fixed domain (which is the classical context of homogenization) our main result is Theorem 3.9 (see also Rems. 3.11 and 3.12). When the domain has an oscillating boundary but the coefficients are fixed, Theorem 5.3 gives a similar result (see also Rem. 5.4). Note that in this latter case, Theorem 5.3 was previously obtained in [1-4,20]. Also higher order error estimates were obtained in [21] (which further considers the case of a three-scale oscillating boundary). Combining these two settings (i.e. homogenization in an oscillating domain) we obtain our main result Theorem 6.8 (see also Rem. 6.9).

The paper is organized as follows. Sections 2 and 3 are devoted to periodic homogenization in a fixed domain. Section 2 is a brief review of the usual homogenization procedure, while Section 3 focus on boundary layers in the particular case of a rectangular domain. The remaining sections are concerned with periodic homogenization in a varying domain with a periodically oscillating boundary. Section 4 gives a precise description of our setting and preliminary technical results. Section 5 investigates the simpler case of constant coefficients, while Section 6 addresses the general case.

\section{Homogenization IN A FIXED DOMAIN}

The goal of this section is to revisit the classical results of homogenization for periodic media and to emphasize the role of the boundary layers. We begin by specifying our notations. Throughout this paper, the letter $C$ denotes a positive constant, the value of which may vary from place to place. If it is not otherwise specified, we adopt the convention that repeated indices indicate summation. Let $\Omega$ be a bounded open subset of $\mathbb{R}^{n}$ with $n \geq 1$. Let $Y=(0,1)^{n}$ be the unit cell in $\mathbb{R}^{n}$. A function $f(x)$, defined on $\mathbb{R}^{n}$, is said to be $Y$-periodic if it is periodic of period 1 with respect to each variable $x_{i}$, with $1 \leq i \leq n$. We denote by $L_{\#}^{2}(Y)$ and $H_{\#}^{1}(Y)$ the spaces of functions in $L_{l o c}^{2}\left(\mathbb{R}^{n}\right)$ and $H_{l o c}^{1}\left(\mathbb{R}^{n}\right)$, respectively, which are $Y$-periodic.

Let $A(y)$ be a square matrix of order $n$ (not necessarily symmetric) with entries $a_{i j}(y)$ which are measurable $Y$-periodic functions. We assume that there exist two constants $0<\lambda<\Lambda<+\infty$ such that, for a.e. $y \in Y$,

$$
\lambda|\xi|^{2} \leq a_{i j}(y) \xi_{i} \xi_{j} \leq \Lambda|\xi|^{2} \quad \forall \xi \in \mathbb{R}^{n} .
$$


Let $A_{\varepsilon}(x)=A\left(\frac{x}{\varepsilon}\right)$ be a periodically oscillating matrix of coefficients. For a given function $f \in L^{2}(\Omega)$ we consider the following equation

$$
\left\{\begin{array}{cl}
-\operatorname{div} A_{\varepsilon} \nabla u_{\varepsilon}=f & \text { in } \Omega, \\
u_{\varepsilon}=0 & \text { on } \partial \Omega,
\end{array}\right.
$$

which admits a unique solution in $H_{0}^{1}(\Omega)$. The homogenization of equation $(2.2)$ is by now a classical matter (see e.g. $[10,11,13,27]$ ). We briefly recall the main ingredients of this process that we shall use later (we mainly follow the exposition of [13], Ch. I §2). The solution $u_{\varepsilon}$ is assumed to admit the following ansatz (or asymptotic expansion)

$$
u_{\varepsilon}(x)=u_{0}\left(x, \frac{x}{\varepsilon}\right)+\varepsilon u_{1}\left(x, \frac{x}{\varepsilon}\right)+\varepsilon^{2} u_{2}\left(x, \frac{x}{\varepsilon}\right)+\varepsilon^{3} u_{3}\left(x, \frac{x}{\varepsilon}\right)+\ldots
$$

where each function $u_{i}(x, y)$ is $Y$-periodic with respect to the fast variable $y$. Plugging this ansatz in equation (2.2) and identifying different powers of $\varepsilon$ yields a cascade of equations. Defining an operator $L_{\varepsilon}$ by $L_{\varepsilon} \phi=$ $-\operatorname{div} A_{\varepsilon} \nabla \phi$, we may write $L_{\varepsilon}=\varepsilon^{-2} L_{0}+\varepsilon^{-1} L_{1}+L_{2}$, where

$$
\begin{aligned}
L_{0} & =-\frac{\partial}{\partial y_{i}}\left(a_{i j}(y) \frac{\partial}{\partial y_{j}}\right) \\
L_{1} & =-\frac{\partial}{\partial y_{i}}\left(a_{i j}(y) \frac{\partial}{\partial x_{j}}\right)-\frac{\partial}{\partial x_{i}}\left(a_{i j}(y) \frac{\partial}{\partial y_{j}}\right) \\
L_{2} & =-\frac{\partial}{\partial x_{i}}\left(a_{i j}(y) \frac{\partial}{\partial x_{j}}\right) .
\end{aligned}
$$

The two space variables $x$ and $y$ are taken as independent, and at the end of the computation $y$ is replaced by $\frac{x}{\varepsilon}$. Equation (2.2) is therefore equivalent to the following system

$$
\begin{aligned}
& L_{0} u_{0}=0 \\
& L_{0} u_{1}+L_{1} u_{0}=0 \\
& L_{0} u_{2}+L_{1} u_{1}+L_{2} u_{0}=f \\
& L_{0} u_{3}+L_{1} u_{2}+L_{2} u_{1}=0 \\
& \ldots \ldots
\end{aligned}
$$

the solutions of which are easily computed. The first equation in (2.4) implies that $u_{0}(x, y) \equiv u(x)$ does not depend on $y$. The second equation of (2.4) gives the value of $u_{1}$ in terms of $u$

$$
u_{1}\left(x, \frac{x}{\varepsilon}\right)=-\chi^{j}\left(\frac{x}{\varepsilon}\right) \frac{\partial u}{\partial x_{j}}(x)+\widetilde{u}_{1}(x)
$$

where $\chi^{j}(y), j=1, \ldots, n$, are the unique solutions in $H_{\#}^{1}(Y)$ with zero average of the cell equation

$$
\begin{cases}L_{0} \chi^{j}=-\frac{\partial a_{i j}}{\partial y_{i}}(y) & \text { in } Y \\ \int_{Y} \chi^{j}(y) d y=0 & y \rightarrow \chi^{j}(y) Y \text {-periodic. }\end{cases}
$$

The third equation in (2.4) gives $u_{2}$ in terms of $u$

$$
u_{2}\left(x, \frac{x}{\varepsilon}\right)=\chi^{i j}\left(\frac{x}{\varepsilon}\right) \frac{\partial^{2} u}{\partial x_{i} \partial x_{j}}(x)-\chi^{j}\left(\frac{x}{\varepsilon}\right) \frac{\partial \widetilde{u}_{1}}{\partial x_{j}}(x)+\widetilde{u}_{2}(x)
$$


where $\chi^{i j} \in H_{\#}^{1}(Y)$, for $i, j=1, \ldots, n$, are the solutions of another cell problem (see [13], (2.42) and (2.39))

$$
\begin{cases}L_{0} \chi^{i j}=b_{i j}-\int_{Y} b_{i j}(y) d y & \text { in } Y \\ \int_{Y} \chi^{i j}(y) d y=0 & y \rightarrow \chi^{i j}(y) Y \text {-periodic }\end{cases}
$$

with

$$
b_{i j}(y)=a_{i j}(y)-a_{i k}(y) \frac{\partial \chi^{j}}{\partial y_{k}}-\frac{\partial}{\partial y_{k}}\left(a_{k i}(y) \chi^{j}\right) .
$$

The homogenized equation for $u(x)$ is obtained by writing the compatibility condition (or Fredholm alternative) for the third equation of (2.4). More precisely, it reads

$$
\left\{\begin{array}{cl}
-\operatorname{div} A^{*} \nabla u=f & \text { in } \Omega \\
u=0 & \text { on } \partial \Omega
\end{array}\right.
$$

where the homogenized matrix $A^{*}$ is defined by its constant entries $a_{i j}^{*}$ given by

$$
a_{i j}^{*}=\int_{Y}\left[a_{i j}(y)-a_{i k}(y) \frac{\partial \chi^{j}}{\partial y_{k}}(y)\right] d y .
$$

Equation (2.9) is well-posed in $H_{0}^{1}(\Omega)$ since it is easily seen that $A^{*}$ is bounded and coercive ([13], Rem. 2.6). Remark that, so far (i.e. if we do not look at higher order Eqs. in (2.4)), the functions $\widetilde{u}_{1}$ in (2.5) and $\widetilde{u}_{2}$ in $(2.7)$ are non-oscillating functions that are not determined. As pointed out in [13], if we stop expansion (2.3) at the first order, the function $\widetilde{u}_{1}$ (and a fortiori $\widetilde{u}_{2}$ ) does not play any role, and so we may choose $\widetilde{u}_{1} \equiv 0$. However, if higher order terms are considered, then $\widetilde{u}_{1}$ must satisfy some equation. More precisely, the compatibility condition of the fourth equation of (2.4) leads to (see [13], Eq. (2.45))

$$
-\operatorname{div} A^{*} \nabla \widetilde{u}_{1}=c_{i j k} \frac{\partial^{3} u}{\partial x_{i} \partial x_{j} \partial x_{k}}
$$

with

$$
c_{i j k}=\int_{Y}\left[a_{k l}(y) \frac{\partial \chi^{i j}}{\partial y_{l}}(y)-a_{i j}(y) \chi^{k}(y)\right] d y .
$$

Similar considerations hold for $\widetilde{u}_{2}$, but we shall not need it in the sequel. Remark that we have not found, so far, any boundary conditions for (2.10). This important question will be answered later on. At this point we emphasize that the above method of two-scale asymptotic expansion is formal. One reason for this is that the ansatz (2.3) is not correct since it does not include boundary layer terms. However, a well-known theorem states that the two first terms of (2.3) are correct.

Theorem 2.1. Let $u_{\varepsilon}$ and $u$ be the unique solutions of (2.2) and (2.9) respectively. Then, $u_{\varepsilon}$ converges weakly to $u$ in $H_{0}^{1}(\Omega)$. If furthermore $u \in W^{2, \infty}(\Omega)$, then

$$
\left\|u_{\varepsilon}(x)-u(x)-\varepsilon u_{1}\left(x, \frac{x}{\varepsilon}\right)\right\|_{H^{1}(\Omega)} \leq C \sqrt{\varepsilon}
$$

where $u_{1}$ is given by (2.5).

The proof of Theorem 2.1 is completely standard (see e.g. [13]). Remark that it holds whatever the choice of $\widetilde{u}_{1}$ is, since the term $\varepsilon \widetilde{u}_{1}(x)$ is smaller than $\sqrt{\varepsilon}$ in the $H^{1}(\Omega)$-norm. The error estimate of order $\sqrt{\varepsilon}$ in Theorem 2.1, although generically optimal, is a little surprising since one could expect to get $\varepsilon$ if the next order term in the ansatz was truly $\varepsilon^{2} u_{2}\left(x, \frac{x}{\varepsilon}\right)$. As is well known, this worse-than-expected result is due to the appearance of boundary layers (see $[11,14,24]$ ). 
To improve the asymptotic expansion (2.3), especially near the boundary of $\Omega$, we need to introduce supplementary terms, called boundary layers. More precisely, the postulated ansatz of $u_{\varepsilon}$ is now

$$
\begin{aligned}
u_{\varepsilon}(x)= & u(x)+\varepsilon\left[u_{1}\left(x, \frac{x}{\varepsilon}\right)+u_{1}^{b l, \varepsilon}(x)\right] \\
& +\varepsilon^{2}\left[u_{2}\left(x, \frac{x}{\varepsilon}\right)+u_{2}^{b l, \varepsilon}(x)\right]+\varepsilon^{3}\left[u_{3}\left(x, \frac{x}{\varepsilon}\right)+u_{3}^{b l, \varepsilon}(x)\right]+\ldots
\end{aligned}
$$

where each function $u_{i}^{b l, \varepsilon}$ satisfies

$$
\begin{cases}-\operatorname{div} A_{\varepsilon} \nabla u_{i}^{b l, \varepsilon}=0 & \text { in } \Omega, \\ u_{i}^{b l, \varepsilon}(x)=-u_{i}\left(x, \frac{x}{\varepsilon}\right) & \text { on } \partial \Omega .\end{cases}
$$

The advantage of ansatz (2.11) over (2.3) is that each term $\left(u_{i}+u_{i}^{b l, \varepsilon}\right)$ satisfies a Dirichlet boundary condition. Remark that $u_{i}^{b l, \varepsilon}(x)$ depends heavily on $\varepsilon$, but is not a function of $(x, x / \varepsilon)$, periodic with respect to the second argument. We are interested only in the first boundary layer $u_{1}^{b l, \varepsilon}$ which is equivalently defined by

$$
\begin{cases}-\operatorname{div} A_{\varepsilon} \nabla u_{1}^{b l, \varepsilon}=0 & \text { in } \Omega \\ u_{1}^{b l, \varepsilon}(x)=\chi^{j}\left(\frac{x}{\varepsilon}\right) \frac{\partial u}{\partial x_{j}}(x)-\widetilde{u}_{1}(x) & \text { on } \partial \Omega\end{cases}
$$

With such a boundary layer term, Theorem 2.1 has been improved as follows in [25].

Theorem 2.2. Let $u_{\varepsilon}$ and $u$ be the unique solutions of (2.2) and (2.9) respectively. Assume that $u \in W^{2, \infty}(\Omega)$. Let $u_{1}, u_{1}^{b l, \varepsilon}$ be defined by (2.5) and (2.12). Then

$$
\left\|u_{\varepsilon}(x)-u(x)-\varepsilon u_{1}\left(x, \frac{x}{\varepsilon}\right)-\varepsilon u_{1}^{b l, \varepsilon}(x)\right\|_{H_{0}^{1}(\Omega)} \leq C \varepsilon .
$$

Proof. As in [25], defining $r_{\varepsilon}(x)=\varepsilon^{-1}\left(u_{\varepsilon}(x)-u(x)-\varepsilon u_{1}(x, x / \varepsilon)-\varepsilon u_{1}^{b l, \varepsilon}(x)\right)$, it satisfies

$$
\begin{cases}-\operatorname{div} A_{\varepsilon} \nabla r_{\varepsilon}=\varepsilon^{-1}\left(f+\operatorname{div} A_{\varepsilon} \nabla u\right)+\operatorname{div} A_{\varepsilon} \nabla u_{1} & \text { in } \Omega, \\ r_{\varepsilon}=0 & \text { on } \partial \Omega .\end{cases}
$$

Taking into account system (2.4), for any $\phi \in H_{0}^{1}(\Omega)$, we have

$$
\begin{aligned}
& \left|\int_{\Omega}\left[\frac{1}{\varepsilon}\left(f+\operatorname{div} A_{\varepsilon} \nabla u\right)+\operatorname{div} A_{\varepsilon} \nabla u_{1}\right] \phi d x\right| \\
& =\left|\int_{\Omega}\left[-\frac{1}{\varepsilon} \operatorname{div}_{y} A_{\varepsilon} \nabla_{y} u_{2}\left(x, \frac{x}{\varepsilon}\right)+\operatorname{div}_{x} A_{\varepsilon} \nabla_{x} u_{1}\left(x, \frac{x}{\varepsilon}\right)\right] \phi d x\right| \\
& \quad \leq\left|\int_{\Omega}-\left[\operatorname{div}_{x} A_{\varepsilon} \nabla_{y} u_{2}\left(x, \frac{x}{\varepsilon}\right)+\frac{1}{\varepsilon} \operatorname{div}_{y} A_{\varepsilon} \nabla_{y} u_{2}\left(x, \frac{x}{\varepsilon}\right)\right] \phi d x\right| \\
& \quad+\left|\int_{\Omega}\left[\operatorname{div}_{x} A_{\varepsilon} \nabla_{y} u_{2}\left(x, \frac{x}{\varepsilon}\right)+\operatorname{div}_{x} A_{\varepsilon} \nabla_{x} u_{1}\left(x, \frac{x}{\varepsilon}\right)\right] \phi d x\right| \\
& \quad \leq\left|\int_{\Omega} A_{\varepsilon} \nabla_{y} u_{2}\left(x, \frac{x}{\varepsilon}\right) \nabla \phi d x\right|+C\|\phi\|_{H_{0}^{1}(\Omega)} \leq C\|\phi\|_{H_{0}^{1}(\Omega)} .
\end{aligned}
$$


Passing to the supremum when $\|\phi\|_{H_{0}^{1}(\Omega)}=1$, we obtain that

$$
\left\|r_{\varepsilon}\right\|_{H_{0}^{1}(\Omega)} \leq \frac{1}{\lambda}\left\|\frac{1}{\varepsilon}\left(f+\operatorname{div} A_{\varepsilon} \nabla u\right)+\operatorname{div} A_{\varepsilon} \nabla u_{1}\right\|_{H^{-1}(\Omega)} \leq C,
$$

which implies the desired result.

Remark again that Theorem 2.2 holds for any choice of $\widetilde{u}_{1}$, since the $H^{1}(\Omega)$-norm of $\varepsilon \widetilde{u}_{1}(x)$ is precisely of order $\varepsilon$. In truth, Theorem 2.2 is not satisfactory since the boundary layer $u_{1}^{b l, \varepsilon}$ is not explicit with respect to $\varepsilon$. To find the asymptotic behavior of $u_{1}^{b l, \varepsilon}$, i.e. to homogenize (2.12), is a very difficult problem that has been addressed only for very special domains $\Omega$, namely with boundaries that are hyperplanes (see e.g. [11,14,24,26]). We shall come back to this question in the next section. For the moment our goal is to improve Theorem 2.2 by removing the boundary layer term at the price of getting merely interior estimates. Indeed if $u_{1}^{b l, \varepsilon}$ is really oscillating only near the boundary, one can expect that it does not play any role for interior estimates.

Unfortunately we are not able to achieve our program without further assumptions. Indeed, we obtain optimal interior estimates mainly in two different cases: first, for a general domain $\Omega$ with either the maximum principle or smooth coefficients in order to apply the compactness method of Avellaneda-Lin [8,9], and second, for general $L^{\infty}$-coefficients with a rectangular domain $\Omega$. The later case is the focus of the next section. We now treat the former case.

Namely we assume one of the following assumptions.

(H1) Equation (2.2) is a scalar equation, i.e. its solution $u_{\varepsilon}$ is a real-valued function, and therefore the maximum principle applies.

(H2) The boundary of $\Omega$ is smooth, say $C^{2}$, and the coefficients $a_{i j}(y)$ in equation (2.2) are Hölder continuous, i.e. there exists $\gamma \in(0,1]$ such that

$$
\sup _{x, y \in \mathbb{R}^{n}} \frac{\left|a_{i j}(x)-a_{i j}(y)\right|}{|x-y|^{\gamma}}=\left\|a_{i j}\right\|_{C^{\gamma}\left(\mathbb{R}^{n}\right)}<+\infty \quad \forall i, j=1, \ldots, n .
$$

Therefore the results of Avellaneda-Lin apply (weaker assumptions on the boundary of $\Omega$ are possible).

Theorem 2.3. Let $u_{\varepsilon}$ and $u$ be the unique solutions of (2.2) and (2.9) respectively. Let $u_{1}$ be defined by (2.5). Assume that either hypothesis (H1) or (H2) holds true. Assume also that $u \in W^{3, \infty}(\Omega)$. Then, for any open set $\omega \subset \subset \Omega$ compactly embedded in $\Omega$, there exists a constant $C$, depending on $\omega$ but not on $\varepsilon$, such that

$$
\left\|u_{\varepsilon}(x)-u(x)-\varepsilon u_{1}\left(x, \frac{x}{\varepsilon}\right)\right\|_{H^{1}(\omega)} \leq C \varepsilon .
$$

Remark 2.4. For the sake of clarity all our results are stated on the simple scalar equation (2.2). Therefore, for this particular equation, assumption (H1) always holds. However, for systems of second-order elliptic equations (H1) is usually not satisfied, which explains why (H2) makes sense. Consequently Theorem 2.3 holds true also for systems of equations. Remark also that, in Theorem 2.3, the choice of the additive function $\widetilde{u}_{1}$ in $(2.5)$ does not matter.

In order to prove Theorem 2.3, we need the following preliminary lemma.

Lemma 2.5. For a sequence $\phi_{\varepsilon}$ in $H^{1}(\Omega)$ we define the sequence of solutions $z_{\varepsilon} \in H^{1}(\Omega)$ of

$$
\begin{cases}-\operatorname{div} A_{\varepsilon} \nabla z_{\varepsilon}=0 & \text { in } \Omega \\ z_{\varepsilon}=\phi_{\varepsilon} & \text { on } \partial \Omega\end{cases}
$$


Assume that there exists a constant $C$ such that, either (H1) holds and $\left\|\phi_{\varepsilon}\right\|_{L^{\infty}(\partial \Omega)} \leq C$, or (H2) holds and $\left\|\phi_{\varepsilon}\right\|_{L^{2}(\partial \Omega)} \leq C$. Then, for any open set $\omega \subset \subset \Omega$, there exists a positive constant $C$ such that

$$
\left\|z_{\varepsilon}\right\|_{H^{1}(\omega)} \leq C
$$

Proof. Let $\omega \subset \subset \subset$. Let $\phi$ be a smooth function with compact support in $\Omega$ such that $\phi=1$ in $\omega$. Multiplying the equation by the test function $\phi^{2} z_{\varepsilon}$, we obtain

$$
\int_{\Omega} \phi^{2} A_{\varepsilon} \nabla z_{\varepsilon} \nabla z_{\varepsilon} d x=-2 \int_{\Omega} \phi z_{\varepsilon} A_{\varepsilon} \nabla z_{\varepsilon} \nabla \phi d x
$$

This implies

$$
\left\|\nabla z_{\varepsilon}\right\|_{L^{2}(\omega)} \leq\left\|\phi \nabla z_{\varepsilon}\right\|_{L^{2}(\Omega)} \leq C\left\|z_{\varepsilon} \nabla \phi\right\|_{L^{2}(\Omega)} \leq C\left\|z_{\varepsilon}\right\|_{L^{2}(\Omega)} .
$$

If (H1) holds (i.e. we can use the maximum principle), then $\left\|z_{\varepsilon}\right\|_{L^{2}(\Omega)} \leq C\left\|\phi_{\varepsilon}\right\|_{L^{\infty}(\partial \Omega)}$. In the other case (H2), the compactness method of Avellaneda-Lin ([8], Th. 3), implies that

$$
\left\|z_{\varepsilon}\right\|_{L^{2}(\Omega)} \leq C\left\|\phi_{\varepsilon}\right\|_{L^{2}(\partial \Omega)}
$$

In both cases we deduce that

$$
\left\|z_{\varepsilon}\right\|_{H^{1}(\omega)} \leq C\left\|z_{\varepsilon}\right\|_{L^{2}(\Omega)} \leq C,
$$

which finishes the proof.

We are now in a position to prove Theorem 2.3.

Proof. For $\omega \subset \subset \Omega$, we observe that

$$
\left\|u_{\varepsilon}-u-\varepsilon u_{1}\right\|_{H^{1}(\omega)} \leq\left\|u_{\varepsilon}-u-\varepsilon u_{1}-\varepsilon u_{1}^{b l, \varepsilon}\right\|_{H_{0}^{1}(\Omega)}+\varepsilon\left\|u_{1}^{b l, \varepsilon}\right\|_{H^{1}(\omega)} .
$$

Since $u_{1}(x, x / \varepsilon)$ is a bounded sequence in either $L^{\infty}(\partial \Omega)$ or $L^{2}(\partial \Omega)$, application of Lemma 2.5 yields the desired result.

We believe that Theorem 2.3 holds true even without assumptions (H1) or (H2), but we are unable to prove it. We conclude this section by remarking that, in Theorem 2.3, we must content ourselves with interior estimates since, in general, the $H^{1}(\Omega)$-norm of $u_{1}^{b l, \varepsilon}$ blows up like $\varepsilon^{-1 / 2}$ as stated in the next lemma (this estimate is optimal as proved in Sect. 4).

Lemma 2.6. Let $\phi_{\varepsilon}$ be a sequence of functions in $W^{1, \infty}(\Omega)$ such that

$$
\left\|\phi_{\varepsilon}\right\|_{L^{\infty}(\Omega)} \leq C \quad \text { and } \quad\left\|\nabla \phi_{\varepsilon}\right\|_{L^{\infty}(\Omega)} \leq \frac{C}{\varepsilon} .
$$

Let $z_{\varepsilon} \in H^{1}(\Omega)$ be the solution of

Then it satisfies

$$
\begin{cases}-\operatorname{div} A_{\varepsilon} \nabla z_{\varepsilon}=0 & \text { in } \Omega, \\ z_{\varepsilon}=\phi_{\varepsilon} & \text { on } \partial \Omega .\end{cases}
$$

$$
\left\|z_{\varepsilon}\right\|_{H^{1}(\Omega)} \leq \frac{C}{\sqrt{\varepsilon}}
$$


Proof. Let us define $\widetilde{z}_{\varepsilon}(x)=\phi_{\varepsilon}(x) \exp \left[-\frac{d(x, \partial \Omega)}{\varepsilon}\right]$. By assumptions, we have that $\left\|\widetilde{z}_{\varepsilon}\right\|_{H^{1}(\Omega)} \leq \frac{C}{\sqrt{\varepsilon}}$. Set $\delta_{\varepsilon}=$ $z_{\varepsilon}-\widetilde{z}_{\varepsilon}$. Clearly, $\delta_{\varepsilon} \in H_{0}^{1}(\Omega)$; hence,

$$
\int_{\Omega} A_{\varepsilon} \nabla z_{\varepsilon} \nabla \delta_{\varepsilon} d x=0
$$

By (2.1) and (2.15), it follows

$$
\lambda\left\|\nabla \delta_{\varepsilon}\right\|_{L^{2}(\Omega)}^{2} \leq \int_{\Omega} A_{\varepsilon} \nabla \delta_{\varepsilon} \nabla \delta_{\varepsilon} d x=-\int_{\Omega} A_{\varepsilon} \nabla \widetilde{z}_{\varepsilon} \nabla \delta_{\varepsilon} d x \leq C\left\|\nabla \widetilde{z}_{\varepsilon}\right\|_{L^{2}(\Omega)}\left\|\nabla \delta_{\varepsilon}\right\|_{L^{2}(\Omega)}
$$

This implies that

and hence

$$
\left\|\nabla \delta_{\varepsilon}\right\|_{L^{2}(\Omega)} \leq C\left\|\nabla \widetilde{z}_{\varepsilon}\right\|_{L^{2}(\Omega)} \leq \frac{C}{\sqrt{\varepsilon}}
$$

$$
\left\|z_{\varepsilon}\right\|_{H^{1}(\Omega)} \leq\left\|\delta_{\varepsilon}\right\|_{H_{0}^{1}(\Omega)}+\left\|\widetilde{z}_{\varepsilon}\right\|_{H^{1}(\Omega)} \leq C\left[\left\|\nabla \delta_{\varepsilon}\right\|_{L^{2}(\Omega)}+\frac{1}{\sqrt{\varepsilon}}\right] \leq \frac{C}{\sqrt{\varepsilon}}
$$

\section{The CASe of a ReCtangular domain}

In this section we pursue the analysis of the previous section when the domain $\Omega$ is rectangular. In such a case we are able to build explicitly the boundary layers and obtain better error estimates for the homogenization process. From now on we assume that $\Omega=(0,1)^{n}$ is the unit open cube. We also assume that the sequence of periods $\varepsilon$ is such that $\varepsilon^{-1} \in \mathbb{N}$, so that the domain is always made of the union of entire cells.

The computation of the boundary layers for equation (2.2) starts with the definition of a new type of unit cell which is unbounded in the direction normal to the boundary. Let us define a semi-infinite strip $G=Y^{\prime} \times(-\infty, 0)$ with $Y^{\prime}=(0,1)^{n-1}$. A point $y \in G$ is denoted by $y=\left(y^{\prime}, y_{n}\right)$ with $y^{\prime} \in Y^{\prime}$ and $y_{n} \in(-\infty, 0)$. The boundary of $G$ is divided in two disjoint parts

$$
\partial G=\Gamma \cup \partial G_{\#},
$$

where $\Gamma=Y^{\prime} \times\{0\}$ and $\partial G_{\#}=\partial Y^{\prime} \times(-\infty, 0)$. For each solution $\chi^{j}$ of the cell problem (2.6), we consider the following problem in $G$

$$
\begin{cases}-\operatorname{div} A(y) \nabla \psi^{j}=0 & \text { in } G, \\ \psi^{j}=\chi^{j} & \text { on } \Gamma, \\ y^{\prime} \rightarrow \psi^{j}\left(y^{\prime}, y_{n}\right) & Y^{\prime} \text {-periodic. }\end{cases}
$$

The existence, uniqueness, and behavior at infinity of the solution to problem (3.1) have been studied by many authors $[11,14,24-26]$. We recall their result on the exponential decay at infinity of the solutions (see also Lem. 4.4 for another proof in a more general context). This type of result is often called a Phragmen-Lindelöf estimate or a Saint-Venant principle for (3.1).

Lemma 3.1. There exists a unique solution $\psi^{j}$ of (3.1) in $H_{l o c}^{1}(G)$. Moreover, there exist an exponent $\gamma>0$ and a unique real constant $d^{j}$ such that

$$
e^{-\gamma y_{n}}\left(\psi^{j}(y)-d^{j}\right) \in L^{2}(G), e^{-\gamma y_{n}} \nabla \psi^{j}(y) \in L^{2}(G) .
$$


Given $j=1, \ldots, n$, let $\psi^{j}$ be the solution of problem (3.1) and $d^{j}$ its associated limit at infinity. By translation and rescaling, we define

$$
\psi_{\varepsilon}^{j}\left(x^{\prime}, x_{n}\right)=\psi^{j}\left(\frac{x^{\prime}}{\varepsilon}, \frac{x_{n}-1}{\varepsilon}\right)-d^{j} .
$$

Clearly, $\psi_{\varepsilon}^{j} \in H^{1}(\Omega)$ and satisfies

$$
\begin{cases}-\operatorname{div} A_{\varepsilon} \nabla \psi_{\varepsilon}^{j}=0 & \text { in } \Omega, \\ \psi_{\varepsilon}^{j}=\chi^{j}\left(\frac{x^{\prime}}{\varepsilon}, 0\right)-d^{j} & \text { on } \Gamma^{1}=\partial \Omega \cap\left\{x_{n}=1\right\}, \\ x^{\prime} \rightarrow \psi_{\varepsilon}^{j}\left(x^{\prime}, x_{n}\right) & \varepsilon Y^{\prime} \text {-periodic. }\end{cases}
$$

Let us define

$$
\widetilde{\psi}_{\varepsilon}^{j}\left(x^{\prime}, x_{n}\right)=\psi_{\varepsilon}^{j}\left(x^{\prime}, x_{n}\right) V\left(x^{\prime}, x_{n}\right),
$$

where $V: \Omega \rightarrow \mathbb{R}$ is a function of $W^{1, \infty}(\Omega)$ such that $V=0$ on $\partial \Omega \backslash \Gamma^{1}$. There are many possible choices of such a function: for instance, we may take $V\left(x^{\prime}, x_{n}\right)=W\left(x^{\prime}\right) x_{n}$ where $W\left(x^{\prime}\right)$ is a smooth function with compact support in $(0,1)^{n-1}$.

Lemma 3.2. Let $\widetilde{\psi}_{\varepsilon}^{j}$ be defined by (3.2). Let $w_{\varepsilon}$ be the unique solution in $H^{1}(\Omega)$ of

$$
\begin{cases}-\operatorname{div} A_{\varepsilon} \nabla w_{\varepsilon}=0 & \text { in } \Omega, \\ w_{\varepsilon}(x)=\widetilde{\psi}_{\varepsilon}^{j}(x)=V(x)\left(\chi^{j}\left(\frac{x}{\varepsilon}\right)-d^{j}\right) & \text { on } \partial \Omega .\end{cases}
$$

Then

$$
\left\|w_{\varepsilon}-\widetilde{\psi}_{\varepsilon}^{j}\right\|_{H_{0}^{1}(\Omega)} \leq C \sqrt{\varepsilon} .
$$

As a consequence of Lemma 3.2, we obtain the following estimate for $w_{\varepsilon}$.

Corollary 3.3. Let $w_{\varepsilon}$ be defined as in Lemma 3.2. Then

$$
\begin{aligned}
\left\|w_{\varepsilon}\right\|_{H^{1}(\Omega)} & \leq \frac{C}{\sqrt{\varepsilon}}, \\
\left\|w_{\varepsilon}\right\|_{L^{2}(\Omega)} & \leq C \sqrt{\varepsilon},
\end{aligned}
$$

and, for every open set $\omega \subset \subset \Omega$, there exists a constant $C>0$ such that

$$
\left\|w_{\varepsilon}\right\|_{H^{1}(\omega)} \leq C \sqrt{\varepsilon}
$$

The proofs of Lemma 3.2 and Corollary 3.3 are similar to those of Lemma 4.6 and Corollary 4.7 of the next section to which we refer for further details.

Of course, for each face of the cubic domain $\Omega$, there is a different normal direction and a different semiinfinite strip $G$. For $m=1, \ldots, 2 n$, we denote by $\Gamma^{m}$ the $m^{t h}$-side of $\Omega$. For each face $\Gamma^{m}$, we denote by $d_{j}^{m}$, $j=1, \ldots, n$, the constant defined in Lemma 3.1 with $G$ oriented properly with respect to $\Gamma^{m}$.

Let us now consider the behaviour of the boundary layer term $u_{1}^{b l, \varepsilon}$, which is defined by (2.12). It can be decomposed in two terms

$$
u_{1}^{b l, \varepsilon}(x)=v_{1}^{b l, \varepsilon}(x)+w_{1}^{b l, \varepsilon}(x),
$$


where $v_{1}^{b l, \varepsilon}$ satisfies

$$
\left\{\begin{array}{cl}
-\operatorname{div} A_{\varepsilon} \nabla v_{1}^{b l, \varepsilon}=0 & \text { in } \Omega, \\
v_{1}^{b l, \varepsilon}(x)=d_{j}^{m} \frac{\partial u}{\partial x_{j}}(x)-\widetilde{u}_{1}(x) & \text { on } \Gamma^{m}, \text { for } m=1, \ldots, 2 n
\end{array}\right.
$$

and $w_{1}^{b l, \varepsilon}$ satisfies

$$
\left\{\begin{array}{cl}
-\operatorname{div} A_{\varepsilon} \nabla w_{1}^{b l, \varepsilon}=0 & \text { in } \Omega, \\
w_{1}^{b l, \varepsilon}(x)=\left(\chi^{j}\left(\frac{x}{\varepsilon}\right)-d_{j}^{m}\right) \frac{\partial u}{\partial x_{j}}(x) & \text { on } \Gamma^{m}, \text { for } m=1, \ldots, 2 n .
\end{array}\right.
$$

The motivation for such a decomposition is that (3.6) is now a standard problem of homogenization (its boundary data does not oscillate), while the solution of (3.7) converges to zero in the interior of the domain. Remark that, if we assume that $u \in W^{2, \infty}(\Omega)$, since $u=0$ on $\partial \Omega$, the boundary datas for (3.6) and (3.7) belong to $H^{\frac{1}{2}}(\partial \Omega)$. Moreover, every tangential derivative of $u$ is zero, hence in equations (3.6) and (3.7), on every side $\Gamma^{m}, m=1, \ldots, 2 n$, it remains only the corresponding normal derivative of $u$.

Applying the result of the previous section, the homogenization of (3.6) yields

$$
\left\|v_{1}^{b l, \varepsilon}(x)-v_{1}^{b l}(x)-\varepsilon v_{1,1}^{b l}\left(x, \frac{x}{\varepsilon}\right)\right\|_{H^{1}(\Omega)} \leq C \sqrt{\varepsilon},
$$

where $v_{1,1}^{b l}(x, y)=\chi^{j}(y) \frac{\partial v_{1}^{b l}}{\partial x_{j}}(x)$ and $v_{1}^{b l}$ is the solution of

$$
\begin{cases}-\operatorname{div} A^{*} \nabla v_{1}^{b l}=0 & \text { in } \Omega, \\ v_{1}^{b l}(x)=d_{j}^{m} \frac{\partial u}{\partial x_{j}}(x)-\widetilde{u}_{1}(x) & \text { on } \Gamma^{m}, \text { for } m=1, \ldots, 2 n .\end{cases}
$$

On the other hand, as a consequence of Lemmas 3.1, 3.2 and Corollary 3.3, we obtain the following result for equation (3.7).

Lemma 3.4. Assume that $u \in W^{2, \infty}(\Omega)$. Let $w_{1}^{b l, \varepsilon}$ be the solution of (3.7). For every open set $\omega \subset \subset \Omega$, there exists a constant $C>0$ such that

$$
\left\|w_{1}^{b l, \varepsilon}\right\|_{L^{2}(\Omega)} \leq C \sqrt{\varepsilon} \quad \text { and } \quad\left\|w_{1}^{b l, \varepsilon}\right\|_{H^{1}(\omega)} \leq C \sqrt{\varepsilon}
$$

Proof. Let $\omega \subset \subset \Omega$. By linearity we decompose the solution $w_{1}^{b l, \varepsilon}$ of (3.7) into the sum of $2 n$ terms $w_{1, m}^{b l, \varepsilon}$, $m=1, \ldots, 2 n$, which are solutions of

$$
\left\{\begin{aligned}
-\operatorname{div} A_{\varepsilon} \nabla w_{1, m}^{b l, \varepsilon}=0 & & \text { in } \Omega, \\
w_{1, m}^{b l, \varepsilon}=\left(\chi^{j}-d_{j}^{m}\right) \frac{\partial u}{\partial x_{j}} & & \text { on } \Gamma^{m}, \\
w_{1, m}^{b l, \varepsilon}=0 & & \text { on } \partial \Omega \backslash \Gamma^{m} .
\end{aligned}\right.
$$

As noted before, taking into account that on every side $\Gamma^{m}$ the tangential derivative of $u$ is zero, the boundary condition on that side becomes $\left(\chi^{j_{m}}-d_{j_{m}}^{m}\right) \frac{\partial u}{\partial x_{j_{m}}}$, where $j_{m}$ is the label of the space coordinate normal to the $m^{t h}$ side. By Lemma 3.2 and Corollary 3.3, with $w_{\varepsilon}=w_{1, m}^{b l, \varepsilon}$ and $V\left(x^{\prime}, x_{j_{m}}\right)=\frac{\partial u}{\partial x_{j_{m}}}\left(x^{\prime}, 1\right) x_{j_{m}}$ if $\Gamma^{m}=\partial \Omega \cap\left\{x_{j_{m}}=1\right\}$ or $V\left(x^{\prime}, x_{j_{m}}\right)=\frac{\partial u}{\partial x_{j_{m}}}\left(x^{\prime}, 0\right)\left(1-x_{j_{m}}\right)$ if $\Gamma^{m}=\partial \Omega \cap\left\{x_{j_{m}}=0\right\}$, we obtain the desired result. 
Theorem 3.5. Let $u_{\varepsilon}$ and $u$ be the unique solutions of (2.2) and (2.9) respectively. Assume that $u \in W^{2, \infty}(\Omega)$. Then, for any open set $\omega \subset \subset \Omega$ compactly embedded in $\Omega$, there exists a constant $C$, depending on $\omega$ but not on $\varepsilon$, such that

$$
\left\|u_{\varepsilon}(x)-u(x)-\varepsilon u_{1}\left(x, \frac{x}{\varepsilon}\right)\right\|_{H^{1}(\omega)} \leq C \varepsilon .
$$

Remark 3.6. The error estimate provided by Theorem 3.5 is the same as that of Theorem 2.3 , but it does not use the same assumptions. In particular, Theorem 3.5 does not require the coefficients to be smooth, and it holds true also for systems of equations (of course, the price to pay is the geometric assumption of a cubic domain). Remark also that it is valid for any choice of the additive function $\widetilde{u}_{1}$ defined in (2.5). Although the proof of Theorem 3.5 uses some informations on the boundary layers, the resulting error estimate does not include any boundary layers.

Proof. Since $v_{1}^{b l, \varepsilon}$ is uniformly bounded in $H^{1}(\Omega)$, and by application of Lemma 3.4, we deduce that, for any open set $\omega \subset \subset \Omega$, we have

$$
\left\|u_{1}^{b l, \varepsilon}\right\|_{H^{1}(\omega)} \leq\left\|v_{1}^{b l, \varepsilon}\right\|_{H^{1}(\Omega)}+\left\|w_{1}^{b l, \varepsilon}\right\|_{H^{1}(\omega)} \leq C .
$$

On the other hand, we have

$$
\left\|u_{\varepsilon}-u-\varepsilon u_{1}\right\|_{H^{1}(\omega)} \leq\left\|u_{\varepsilon}-u-\varepsilon u_{1}-\varepsilon u_{1}^{b l, \varepsilon}\right\|_{H_{0}^{1}(\Omega)}+\varepsilon\left\|u_{1}^{b l, \varepsilon}\right\|_{H^{1}(\omega)},
$$

which, combined with Theorem 2.2, yields the desired estimate.

Using more deeply our knowledge of the boundary layers (at least in the case of rectangular domains), Theorem 3.5 can be improved as follows.

Theorem 3.7. Let $u_{\varepsilon}$ and $u$ be the unique solutions of (2.2) and (2.9) respectively. Assume that $u \in W^{3, \infty}(\Omega)$. Let $u_{1}$ be defined by (2.5) and assume that $\widetilde{u}_{1}$ satisfies equation (2.10). Then,

$$
\left\|u_{\varepsilon}(x)-u(x)-\varepsilon u_{1}\left(x, \frac{x}{\varepsilon}\right)-\varepsilon u_{1}^{b l, \varepsilon}(x)-\varepsilon^{2} u_{2}\left(x, \frac{x}{\varepsilon}\right)\right\|_{H^{1}(\Omega)} \leq C \varepsilon^{\frac{3}{2}} .
$$

Furthermore, for any open set $\omega \subset \subset \Omega$ compactly embedded in $\Omega$, there exists a constant $C$, independent of $\varepsilon$, such that

$$
\left\|u_{\varepsilon}(x)-u(x)-\varepsilon u_{1}\left(x, \frac{x}{\varepsilon}\right)-\varepsilon v_{1}^{b l}(x)-\varepsilon^{2} v_{1,1}^{b l}\left(x, \frac{x}{\varepsilon}\right)-\varepsilon^{2} u_{2}\left(x, \frac{x}{\varepsilon}\right)\right\|_{H^{1}(\omega)} \leq C \varepsilon^{\frac{3}{2}},
$$

and

$$
\left\|u_{\varepsilon}(x)-u(x)-\varepsilon u_{1}\left(x, \frac{x}{\varepsilon}\right)-\varepsilon v_{1}^{b l}(x)\right\|_{L^{2}(\omega)} \leq C \varepsilon^{\frac{3}{2}} .
$$

Remark 3.8. It is essential in Theorem 3.7 that the additive function $\widetilde{u}_{1}$ satisfies a specific equation, namely (2.10), otherwise the results are wrong. However, its boundary condition is not specified so far. Remark also that, on the contrary of (3.11), each term in (3.12) and (3.13) has an explicit dependence with respect to $\varepsilon$.

Proof. Let $\omega \subset \subset \Omega$. Since $u_{1}^{b l, \varepsilon}=v_{1}^{b l, \varepsilon}+w_{1}^{b l, \varepsilon}$, we observe that

$$
\begin{aligned}
& \left\|u_{\varepsilon}-u-\varepsilon u_{1}-\varepsilon v_{1}^{b l}-\varepsilon^{2} v_{1,1}^{b l}-\varepsilon^{2} u_{2}\right\|_{H^{1}(\omega)} \\
& \leq\left\|u_{\varepsilon}-u-\varepsilon u_{1}-\varepsilon u_{1}^{b l, \varepsilon}-\varepsilon^{2} u_{2}\right\|_{H^{1}(\Omega)}+\varepsilon\left\|w_{1}^{b l, \varepsilon}\right\|_{H^{1}(\omega)}+\varepsilon\left\|v_{1}^{b l, \varepsilon}-v_{1}^{b l}-\varepsilon v_{1,1}^{b l}\right\|_{H^{1}(\Omega)} .
\end{aligned}
$$


By Lemma 3.4, the second term in the previous inequality is bounded by $C \varepsilon^{\frac{3}{2}}$, while, by (3.8), the third one is bounded by $C \varepsilon^{\frac{3}{2}}$. Hence, it remains to estimate the first term. We proceed as in the proof of Theorem 2.3. Defining

it satisfies

$$
r_{\varepsilon}(x)=\frac{u_{\varepsilon}(x)-u(x)-\varepsilon u_{1}\left(x, \frac{x}{\varepsilon}\right)-\varepsilon u_{1}^{b l, \varepsilon}(x)-\varepsilon^{2} u_{2}\left(x, \frac{x}{\varepsilon}\right)}{\varepsilon^{2}},
$$

$$
\begin{cases}-\operatorname{div} A_{\varepsilon} \nabla r_{\varepsilon}=\frac{1}{\varepsilon^{2}}\left(f+\operatorname{div} A_{\varepsilon} \nabla u\right)+\frac{1}{\varepsilon} \operatorname{div} A_{\varepsilon} \nabla u_{1}+\operatorname{div} A_{\varepsilon} \nabla u_{2} & \text { in } \Omega, \\ r_{\varepsilon}(x)=-u_{2}\left(x, \frac{x}{\varepsilon}\right) & \text { on } \partial \Omega .\end{cases}
$$

We decompose $r_{\varepsilon}=r_{\varepsilon}^{1}+r_{\varepsilon}^{2}$, where $r_{\varepsilon}^{1}$ satisfies

$$
\begin{cases}-\operatorname{div} A_{\varepsilon} \nabla r_{\varepsilon}^{1}=\frac{1}{\varepsilon^{2}}\left(f+\operatorname{div} A_{\varepsilon} \nabla u\right)+\frac{1}{\varepsilon} \operatorname{div} A_{\varepsilon} \nabla u_{1}+\operatorname{div} A_{\varepsilon} \nabla u_{2} & \text { in } \Omega \\ r_{\varepsilon}^{1}=0 & \text { on } \partial \Omega\end{cases}
$$

and $r_{\varepsilon}^{2}$ satisfies

$$
\begin{cases}-\operatorname{div} A_{\varepsilon} \nabla r_{\varepsilon}^{2}=0 & \text { in } \Omega \\ r_{\varepsilon}^{2}(x)=-u_{2}\left(x, \frac{x}{\varepsilon}\right)=-\chi^{i j}\left(\frac{x}{\varepsilon}\right) \frac{\partial^{2} u}{\partial x_{i} \partial x_{j}}(x)+\chi^{j}\left(\frac{x}{\varepsilon}\right) \frac{\partial \widetilde{u}_{1}}{\partial x_{j}}(x)-\widetilde{u}_{2}(x) & \text { on } \partial \Omega\end{cases}
$$

Since

$$
\left\|u_{2}\right\|_{L^{\infty}(\Omega)} \leq C \quad \text { and } \quad\left\|\nabla u_{2}\right\|_{L^{\infty}(\Omega)} \leq \frac{C}{\varepsilon}
$$

by Lemma 2.6, it follows that $\left\|r_{\varepsilon}^{2}\right\|_{H^{1}(\Omega)} \leq \frac{C}{\sqrt{\varepsilon}}$. On the other hand, we have

$$
\left\|r_{\varepsilon}^{1}\right\|_{H_{0}^{1}(\Omega)} \leq \frac{1}{\lambda}\left\|\frac{1}{\varepsilon^{2}}\left(f+\operatorname{div} A_{\varepsilon} \nabla u\right)+\frac{1}{\varepsilon} \operatorname{div} A_{\varepsilon} \nabla u_{1}+\operatorname{div} A_{\varepsilon} \nabla u_{2}\right\|_{H^{-1}(\Omega)} .
$$

Using the cascade of equations (2.4) (including Eq. (2.10) for $\widetilde{u}_{1}$ ), for any $\phi \in H_{0}^{1}(\Omega)$, we have

$$
\begin{aligned}
& \left|\int_{\Omega}\left[\frac{1}{\varepsilon^{2}}\left(f+\operatorname{div} A_{\varepsilon} \nabla u\right)+\frac{1}{\varepsilon} \operatorname{div} A_{\varepsilon} \nabla u_{1}+\operatorname{div} A_{\varepsilon} \nabla u_{2}\right] \phi d x\right| \\
& =\left|\int_{\Omega}\left[-\frac{1}{\varepsilon}\left(\operatorname{div}_{y} A \nabla_{y} u_{3}\right)\left(x, \frac{x}{\varepsilon}\right)+\left(\operatorname{div}_{x} A \nabla_{x} u_{2}\right)\left(x, \frac{x}{\varepsilon}\right)\right] \phi d x\right| \\
& \leq\left|\int_{\Omega}-\left[\left(\operatorname{div}_{x} A \nabla_{y} u_{3}\right)\left(x, \frac{x}{\varepsilon}\right)+\frac{1}{\varepsilon}\left(\operatorname{div}_{y} A \nabla_{y} u_{3}\right)\left(x, \frac{x}{\varepsilon}\right)\right] \phi d x\right| \\
& +\left|\int_{\Omega}\left[\operatorname{div}_{x} A_{\varepsilon} \nabla_{y} u_{3}\left(x, \frac{x}{\varepsilon}\right)+\operatorname{div}_{x} A_{\varepsilon} \nabla_{x} u_{2}\left(x, \frac{x}{\varepsilon}\right)\right] \phi d x\right| \\
& \leq\left|\int_{\Omega}\left(A \nabla_{y} u_{3}\right)\left(x, \frac{x}{\varepsilon}\right) \nabla \phi d x\right|+C\|\phi\|_{H_{0}^{1}(\Omega)} \leq C\|\phi\|_{H_{0}^{1}(\Omega)} .
\end{aligned}
$$

Passing to the supremum when $\|\phi\|_{H_{0}^{1}(\Omega)}=1$, we obtain

$$
\left\|r_{\varepsilon}^{1}\right\|_{H_{0}^{1}(\Omega)} \leq C\left\|\frac{1}{\varepsilon^{2}}\left(f+\operatorname{div} A_{\varepsilon} \nabla u\right)+\frac{1}{\varepsilon} \operatorname{div} A_{\varepsilon} \nabla u_{1}+\operatorname{div} A_{\varepsilon} \nabla u_{2}\right\|_{H^{-1}(\Omega)} \leq C .
$$

Finally, we get $\varepsilon^{2}\left\|r_{\varepsilon}\right\|_{H^{1}(\Omega)} \leq C \varepsilon^{\frac{3}{2}}$ which is the desired result. 
If we are interested only in interior estimates as (3.12) and (3.13) in Theorem 3.7, we can get rid of all boundary layers by a clever choice of the additive function $\widetilde{u}_{1}$ and obtain the main result of this section.

Theorem 3.9. Let $u_{\varepsilon}$ and $u$ be the unique solutions of (2.2) and (2.9) respectively. Assume that $u \in W^{3, \infty}(\Omega)$. Let $u_{1}$ be defined by

where $\widetilde{u}_{1}$ is the unique solution of

$$
u_{1}\left(x, \frac{x}{\varepsilon}\right)=-\chi^{j}\left(\frac{x}{\varepsilon}\right) \frac{\partial u}{\partial x_{j}}(x)+\widetilde{u}_{1}(x),
$$

$$
\begin{cases}-\operatorname{div} A^{*} \nabla \widetilde{u}_{1}=c_{i j k} \frac{\partial^{3} u}{\partial x_{i} \partial x_{j} \partial x_{k}} & \text { in } \Omega, \\ \widetilde{u}_{1}=d_{j}^{m} \frac{\partial u}{\partial x_{j}} & \text { on } \Gamma^{m} \text { for } m=1, \ldots, 2 n,\end{cases}
$$

with

$$
c_{i j k}=\int_{Y}\left[a_{k l}(y) \frac{\partial \chi^{i j}}{\partial y_{l}}(y)-a_{i j}(y) \chi^{k}(y)\right] d y .
$$

Then, for any open set $\omega \subset \subset \Omega$ compactly embedded in $\Omega$, there exists a constant $C$, depending on $\omega$ but not on $\varepsilon$, such that

and

$$
\left\|u_{\varepsilon}(x)-u(x)-\varepsilon u_{1}\left(x, \frac{x}{\varepsilon}\right)-\varepsilon^{2} u_{2}\left(x, \frac{x}{\varepsilon}\right)\right\|_{H^{1}(\omega)} \leq C \varepsilon^{\frac{3}{2}}
$$

$$
\left\|u_{\varepsilon}(x)-u(x)-\varepsilon u_{1}\left(x, \frac{x}{\varepsilon}\right)\right\|_{L^{2}(\omega)} \leq C \varepsilon^{\frac{3}{2}} .
$$

Proof. To eliminate the boundary layer, i.e. to have $v_{1}^{b l, \varepsilon}=0$, and therefore $v_{1}^{b l}=v_{1,1}^{b l}=0$, it is enough to choose as boundary condition for $\widetilde{u}_{1}$ (see (3.6) and (3.9))

$$
\widetilde{u}_{1}=d_{j}^{m} \frac{\partial u}{\partial x_{j}} \quad \text { on } \Gamma^{m}, \text { for } m=1, \ldots, 2 n .
$$

Together with (2.10), this yields equation (3.14) which uniquely determines $\widetilde{u}_{1}$. Remark that, $u$ being smooth enough, the boundary data for $\widetilde{u}_{1}$ belongs to $H^{\frac{1}{2}}(\partial \Omega)$. Then, with zero boundary layers Theorem 3.7 yields the desired result.

Remark 3.10. Theorem 3.9 shows that choosing appropriately the boundary condition for $\widetilde{u}_{1}$ cancels out boundary layers in interior estimates. Remark however that boundary layers still play a role through their limit values $d_{j}^{m}$ which appear in the boundary condition for $\widetilde{u}_{1}$. On the contrary, for any other choice of $\widetilde{u}_{1}$, boundary layers appear explicitly in error estimates. Theorems 3.7 and 3.9 hold true also for systems of equations.

Remark 3.11. In many practical applications of homogenization corrector terms are needed in order to improve the quality of approximation of the true unknown $u_{\varepsilon}$ by the homogenized one $u$. However, most of the corrector results are given in the $H^{1}(\Omega)$-norm while sometimes a physically meaningfull estimate is rather stated in the $L^{2}(\Omega)$-norm. If boundary layers are not introduced, then Theorem 3.5 yields a deceptive $L^{2}(\Omega)$-norm estimate

$$
\left\|u_{\varepsilon}(x)-u(x)\right\|_{L^{2}(\omega)} \leq C \varepsilon
$$

since the corrector $u_{1}$ is not used. However, pushing further the analysis, Theorem 3.9 leads to a much better $L^{2}(\Omega)$-norm estimate

$$
\left\|u_{\varepsilon}(x)-u(x)-\varepsilon \chi^{j}\left(\frac{x}{\varepsilon}\right) \frac{\partial u}{\partial x_{j}}(x)-\varepsilon \widetilde{u}_{1}(x)\right\|_{L^{2}(\omega)} \leq C \varepsilon^{\frac{3}{2}}
$$


where remarkably it is not necessary to compute the higher order corrector $u_{2}$ but simply the additive term $\widetilde{u}_{1}$, solution of (3.14). All the information on boundary layer tails is concentrated in the $\widetilde{u}_{1}$ term. To see what effects it has on the homogenized limit, it is instructive to introduce a new function $\bar{u}_{\varepsilon}$ defined by

$$
\bar{u}_{\varepsilon}(x)=u(x)+\varepsilon \widetilde{u}_{1}(x) .
$$

Then, formally $\bar{u}_{\varepsilon}$ is a solution of

$$
\begin{cases}-\operatorname{div} A^{*} \nabla \bar{u}_{\varepsilon}-\varepsilon c_{i j k} \frac{\partial^{3} \bar{u}_{\varepsilon}}{\partial x_{i} \partial x_{j} \partial x_{k}}=f+\mathcal{O}\left(\varepsilon^{2}\right) & \text { in } \Omega, \\ \bar{u}_{\varepsilon}=\varepsilon d_{j}^{m} \frac{\partial \bar{u}_{\varepsilon}}{\partial x_{j}}+\mathcal{O}\left(\varepsilon^{2}\right) & \text { on } \Gamma^{m} \text { for } m=1, \ldots, 2 n,\end{cases}
$$

where $\mathcal{O}\left(\varepsilon^{2}\right)$ denotes remaider terms of order $\varepsilon^{2}$ in both $L^{2}(\Omega)$ and $H^{1}(\Omega)$ norms. Remark that the dependence on $\varepsilon$ in $\bar{u}_{\varepsilon}$ is explicit (on the contrary of $u_{\varepsilon}$ ) and that it is not an oscillating function. The error estimate (3.15) can be rewritten as

$$
\left\|u_{\varepsilon}(x)-\bar{u}_{\varepsilon}(x)-\varepsilon \chi^{j}\left(\frac{x}{\varepsilon}\right) \frac{\partial u}{\partial x_{j}}(x)\right\|_{L^{2}(\omega)} \leq C \varepsilon^{\frac{3}{2}} .
$$

Clearly, equation (3.16) displays dispersive effects and an effective Fourier boundary condition as a consequence of the boundary layers tail. This dispersive effect of homogenization was already recognized in previous works $[13,28]$. The Fourier boundary condition is reminiscent of the so-called albedo condition in neutronics and $\varepsilon d_{j}^{m}$ appears as an extrapolation length (see e.g. Chaps. 19, 21). Remark that, if we cancel the small $\varepsilon^{2}$-order terms in (3.16), the existence of a solution for this dispersive equation is unclear (in particular it lacks a supplementary boundary condition). Nevertheless, from a computational point of view, for small $\varepsilon$, one can avoid the separate computations of $u$ and $\widetilde{u}_{1}$, by computing directly $\bar{u}_{\varepsilon}$ solution of (3.16). Note also that the constants $d_{j}^{m}$, related to the boundary layer tails, can either be computed exactly, or they can be fitted a posteriori to minimize the numerical error for some test cases.

Remark 3.12. When the matrix of coefficients $A(y)$ has a cubic symmetry (i.e. is an even function with respect to the center of the unit cube $Y$ ), it turns out that Theorem 3.9 simplifies considerably since, in this case, $\widetilde{u}_{1}$ is identically equal to 0 . Indeed, under this cubic symmetry assumption, one can easily checked that the functions $\chi^{j}(y)$, defined by (2.6), and $\chi^{i j}(y)$, defined by (2.8), inherit special odd and even properties which implies that the dispersive third-order tensor $c_{i j k}$ is equal to zero. Moreover, $\chi^{j}(y)$ satisfies a Dirichlet boundary conditions on that part of $\partial Y$ which is normal to direction $e_{j}$. Therefore, for such $j$ 's, the boundary layers $\psi^{j}(y)$, defined by (3.1), are identically 0 , as well as their limit values at infinity $d_{j}^{m}$. In other words, there is no dispersive effect and boundary layers (at first order in $\varepsilon$ ) for periodic media with symmetric cells.

\section{Homogenization in AN Oscillating DOMAin}

This section is devoted to a generalization of the results of the previous section when the homogenization process takes place in a domain with a periodically oscillating boundary. We begin by defining a domain $\Omega_{\varepsilon}$ which is a rectangular domain having one face which is periodically oscillating with a period $\varepsilon$. The period $\varepsilon$ is a positive real number such that $\varepsilon^{-1} \in \mathbb{N}$. Denoting by $Y^{\prime}=(0,1)^{n-1}$ the unit cube in $\mathbb{R}^{n-1}$, we introduce a profile function $g: \mathbb{R}^{n-1} \rightarrow \mathbb{R}$ that will define the oscillating boundary of $\Omega_{\varepsilon}$. We assume that $g \in W^{1, \infty}\left(Y^{\prime}\right)$ is an $Y^{\prime}$-periodic function satisfying, for all $y^{\prime} \in Y^{\prime}$,

$$
0 \leq g\left(y^{\prime}\right) \leq 1 \quad \text { and } \quad\left|\nabla g\left(y^{\prime}\right)\right| \leq 1
$$


$\Gamma_{\varepsilon}$

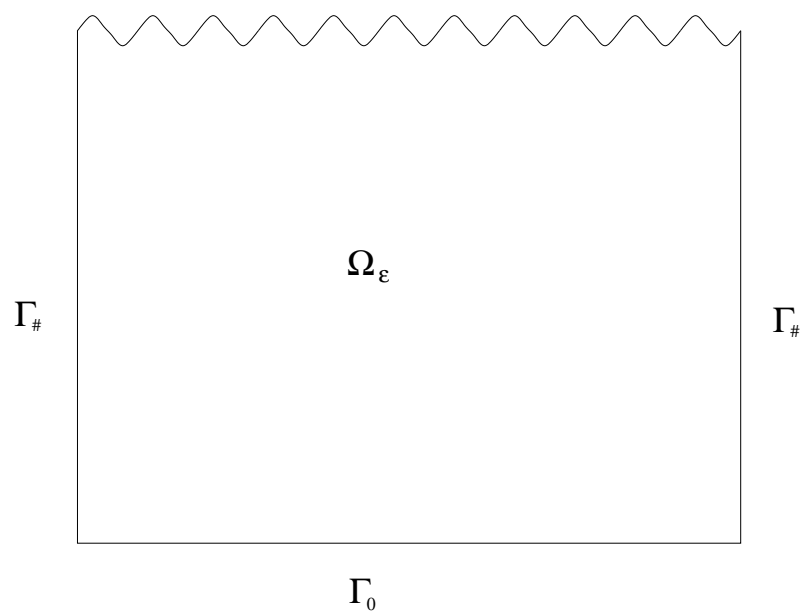

FiguRE 1. The set $\Omega_{\varepsilon}$.

Upon rescaling to size $\varepsilon$, we define a function $g_{\varepsilon}: \mathbb{R}^{n-1} \rightarrow \mathbb{R}$ by

$$
g_{\varepsilon}\left(x^{\prime}\right)=1+\varepsilon\left(g\left(\frac{x^{\prime}}{\varepsilon}\right)-1\right)
$$

which satisfies $1-\varepsilon \leq g_{\varepsilon}\left(x^{\prime}\right) \leq 1$ and $\left|\nabla g_{\varepsilon}\left(x^{\prime}\right)\right| \leq 1$. Then, the oscillating domain $\Omega_{\varepsilon}$ is defined by (see Fig. 1)

$$
\Omega_{\varepsilon}=\left\{x=\left(x^{\prime}, x_{n}\right) \in \mathbb{R}^{n}: x^{\prime} \in(0,1)^{n-1}, 0<x_{n}<g_{\varepsilon}\left(x^{\prime}\right)\right\},
$$

and its oscillating boundary $\Gamma_{\varepsilon}$

$$
\Gamma_{\varepsilon}=\left\{x=\left(x^{\prime}, x_{n}\right) \in \mathbb{R}^{n}: x^{\prime} \in[0,1]^{n-1}, x_{n}=g_{\varepsilon}\left(x^{\prime}\right)\right\} .
$$

Clearly, as $\varepsilon$ goes to $0, \Omega_{\varepsilon}$ converges to a limit domain $\Omega=(0,1)^{n}$ and $\Gamma_{\varepsilon}$ to a limit surface $\Gamma=[0,1]^{n-1} \times\{1\}$. We denote by $\Gamma_{0}=[0,1]^{n-1} \times\{0\}$ the opposite face, and by $\Gamma_{\#}=\partial \Omega \backslash\left(\Gamma \cup \Gamma_{0}\right)$ the rest of the boundary of $\Omega$. Our definition of $g_{\varepsilon}$ implies that $\Omega_{\varepsilon}$ stays entirely included in $\Omega$. Similarly $\partial \Omega_{\varepsilon} \backslash \Gamma_{\varepsilon} \subseteq \partial \Omega \backslash \Gamma$, and for the sake of simplicity we still denote by $\Gamma_{\#}$ the lateral boundaries of $\Omega_{\varepsilon}$, i.e. $\partial \Omega_{\varepsilon} \backslash\left(\Gamma_{\varepsilon} \cup \Gamma_{0}\right)$, although it may be a strict subset of $\partial \Omega \backslash\left(\Gamma \cup \Gamma_{0}\right)$.

As in Section 2 we consider a periodically oscillating matrix $A_{\varepsilon}(x)=A\left(\frac{x}{\varepsilon}\right)$ with the same period $\varepsilon$ than the oscillating boundary (the matrix $A(y)$ still satisfies the assumptions of coercivity and boundedness of Sect. 2). Instead of having Dirichlet boundary conditions everywhere, we consider here, and in the sequel, periodic conditions on the lateral boundaries of $\Omega$ and $\Omega_{\varepsilon}$, i.e. on $\Gamma_{\#}$. For a given function $f \in L^{2}(\Omega)$, with $x^{\prime} \rightarrow f\left(x^{\prime}, x_{n}\right)$ periodic, we consider the following equation

$$
\left\{\begin{array}{cl}
-\operatorname{div} A_{\varepsilon} \nabla u_{\varepsilon}=f & \text { in } \Omega_{\varepsilon}, \\
u_{\varepsilon}=0 & \text { on } \Gamma_{\varepsilon} \cup \Gamma_{0}, \\
x^{\prime} \rightarrow u_{\varepsilon}\left(x^{\prime}, x_{n}\right) & \text { periodic on } \Gamma_{\#},
\end{array}\right.
$$

which admits a unique solution in $H\left(\Omega_{\varepsilon}\right)$ defined by

$$
H\left(\Omega_{\varepsilon}\right)=\left\{u \in H^{1}\left(\Omega_{\varepsilon}\right): u=0 \text { on } \Gamma_{\varepsilon} \cup \Gamma_{0}, x^{\prime} \rightarrow u\left(x^{\prime}, x_{n}\right) \text {-periodic on } \Gamma_{\#}\right\} .
$$


We denote by $H^{\prime}\left(\Omega_{\varepsilon}\right)$ the topological dual of $H\left(\Omega_{\varepsilon}\right)$. Analogously, we introduce the space $H(\Omega)=\{u \in$ $H^{1}(\Omega): u=0$ on $\Gamma \cup \Gamma_{0}, x^{\prime} \rightarrow u\left(x^{\prime}, x_{n}\right)$-periodic on $\left.\Gamma_{\#}\right\}$ and its topological dual space $H^{\prime}(\Omega)$. Remark also that, since $\Omega_{\varepsilon} \subset \Omega$, every function $f \in H\left(\Omega_{\varepsilon}\right)$ can be easily extended by 0 in $\Omega \backslash \Omega_{\varepsilon}$ to become a function of $H(\Omega)$.

We choose to have periodic boundary conditions on $\Gamma_{\#}$, instead of Dirichlet ones, for the sake of simplicity. Indeed, this implies that no boundary layers are created on the lateral sides, and hence, we can concentrate on the oscillation of the boundary and its interaction with the oscillation of the operator. In particular, we avoid the study of possible boundary layers at the corners of $\Gamma_{\varepsilon} \cap \Gamma_{\#}$.

Remark that we implicitely assumed that the oscillations of the boundary and of the coefficients have the same frequency, i.e. their period are not only of the same order of magnitude $\varepsilon$ but are equal (or at least commensurable). We suspect that the interaction between these two types of oscillations would be much weaker in the case of different frequencies. However, we have not investigated this problem further.

The homogenization of equation (4.1) can formally be done as in Section 2 (this type of problems with Dirichlet or Neumann boundary conditions has been addressed in $[2,4,16,27])$. As before, the solution $u_{\varepsilon}$ is assumed to admit the following asymptotic expansion which includes boundary layers at all orders

$$
u_{\varepsilon}(x)=u(x)+u_{0}^{b l, \varepsilon}(x)+\varepsilon\left[u_{1}\left(x, \frac{x}{\varepsilon}\right)+u_{1}^{b l, \varepsilon}(x)\right]+\varepsilon^{2}\left[u_{2}\left(x, \frac{x}{\varepsilon}\right)+u_{2}^{b l, \varepsilon}(x)\right]+\ldots
$$

where each boundary layer term $u_{i}^{b l, \varepsilon}$ satisfies

$$
\begin{cases}-\operatorname{div} A_{\varepsilon} \nabla u_{i}^{b l, \varepsilon}=0 & \text { in } \Omega_{\varepsilon}, \\ u_{i}^{b l, \varepsilon}(x)=-u_{i}\left(x, \frac{x}{\varepsilon}\right) & \text { on } \Gamma_{\varepsilon} \cup \Gamma_{0}, \\ x^{\prime} \rightarrow u_{i}^{b l, \varepsilon}\left(x^{\prime}, x_{n}\right) & \text { periodic on } \Gamma_{\#},\end{cases}
$$

with the convention that, for $i=0, u_{0}\left(x, \frac{x}{\varepsilon}\right) \equiv u(x)$.

It turns out that the functions $u(x), u_{1}(x, y), u_{2}(x, y), u_{3}(x, y) \ldots$, satisfy similar problems as in Section 3 (with periodic boundary conditions on the lateral sides). One need also to introduce the additive functions $\widetilde{u}_{1}(x), \widetilde{u}_{2}(x), \ldots$, as in Section 3; the only difference being in the definition of boundary layers (up to the periodic lateral boundary conditions). In the present setting, boundary layers are the consequence of two different facts: first, the boundary $\Gamma_{\varepsilon}$ is oscillating, and second the terms $u_{i}$ with $i \geq 1$ do not satisfy the Dirichlet boundary condition. Remark also that a boundary layer appears already at the zero-th order in expansion (4.2) because $u$ does not satisfy the Dirichlet boundary condition on $\Gamma_{\varepsilon}$.

As before, the zero-th order term $u(x)$ is the solution of the homogenized problem

$$
\left\{\begin{array}{cl}
-\operatorname{div} A^{*} \nabla u=f & \text { in } \Omega, \\
u=0 & \text { on } \Gamma \cup \Gamma_{0}, \\
x^{\prime} \rightarrow u\left(x^{\prime}, x_{n}\right) & \text { periodic on } \Gamma_{\#} .
\end{array}\right.
$$

Upon extending $u_{\varepsilon}$ by 0 in $\Omega \backslash \Omega_{\varepsilon}$, a standard proof (that we omit) leads to the following

Theorem 4.1. Let $u_{\varepsilon}$ be the unique solution in $H\left(\Omega_{\varepsilon}\right)$ of (4.1) and $u$ be the unique solution in $H(\Omega)$ of (4.4). Then the sequence $u_{\varepsilon}$ converges weakly to $u$ in $H(\Omega)$.

In order to simplify the exposition, Section 5 will focus on the case where the operator has constant coefficients, namely $A(y) \equiv I d$. The general case, for which there is an intricate interaction between homogenization and boundary oscillations, is treated in Section 6 . The end of this section is devoted to a series of technical lemmae, that will be used in Sections 5 and 6 .

We begin with a variant of Lemma 2.6 adapted to the periodic boundary conditions on $\Gamma_{\#}$. 
Lemma 4.2. Let $\phi_{\varepsilon}$ be a sequence of functions in $W^{1, \infty}\left(\Omega_{\varepsilon}\right)$ satisfying periodic boundary conditions on $\Gamma_{\#}$ and such that

$$
\left\|\phi_{\varepsilon}\right\|_{L^{\infty}\left(\Omega_{\varepsilon}\right)} \leq C \quad \text { and } \quad\left\|\nabla \phi_{\varepsilon}\right\|_{L^{\infty}\left(\Omega_{\varepsilon}\right)} \leq \frac{C}{\varepsilon} .
$$

Let $z_{\varepsilon}$ be the unique solution in $H^{1}\left(\Omega_{\varepsilon}\right)$ of

$$
\begin{cases}-\operatorname{div} A_{\varepsilon} \nabla z_{\varepsilon}=0 & \text { in } \Omega_{\varepsilon}, \\ z_{\varepsilon}=\phi_{\varepsilon} & \text { on } \Gamma_{\varepsilon} \cup \Gamma_{0}, \\ x^{\prime} \rightarrow z_{\varepsilon}\left(x^{\prime}, x_{n}\right) & \text { periodic on } \Gamma_{\#} .\end{cases}
$$

Then, it satisfies

$$
\left\|z_{\varepsilon}\right\|_{H^{1}\left(\Omega_{\varepsilon}\right)} \leq \frac{C}{\sqrt{\varepsilon}}
$$

Proof. Let us define $\widetilde{z}_{\varepsilon}(x)=\phi_{\varepsilon}(x) \exp \left[-\frac{d\left(x_{n}, \Gamma_{\varepsilon} \cup \Gamma_{0}\right)}{\varepsilon}\right]$. By assumptions, we have that $\left\|\widetilde{z}_{\varepsilon}\right\|_{H^{1}\left(\Omega_{\varepsilon}\right)} \leq \frac{C}{\sqrt{\varepsilon}}$. Set $\delta_{\varepsilon}=z_{\varepsilon}-\widetilde{z}_{\varepsilon}$. Clearly, $\delta_{\varepsilon} \in H\left(\Omega_{\varepsilon}\right)$; hence,

$$
\int_{\Omega_{\varepsilon}} A_{\varepsilon} \nabla z_{\varepsilon} \nabla \delta_{\varepsilon} d x=0
$$

By (2.1) and (4.5), it follows

$$
\lambda\left\|\nabla \delta_{\varepsilon}\right\|_{L^{2}\left(\Omega_{\varepsilon}\right)}^{2} \leq \int_{\Omega_{\varepsilon}} A_{\varepsilon} \nabla \delta_{\varepsilon} \nabla \delta_{\varepsilon} d x=-\int_{\Omega_{\varepsilon}} A_{\varepsilon} \nabla \widetilde{z}_{\varepsilon} \nabla \delta_{\varepsilon} d x \leq C\left\|\nabla \widetilde{z}_{\varepsilon}\right\|_{L^{2}\left(\Omega_{\varepsilon}\right)}\left\|\nabla \delta_{\varepsilon}\right\|_{L^{2}\left(\Omega_{\varepsilon}\right)} .
$$

This implies that

and hence

$$
\left\|\nabla \delta_{\varepsilon}\right\|_{L^{2}(\Omega)}=\left\|\nabla \delta_{\varepsilon}\right\|_{L^{2}\left(\Omega_{\varepsilon}\right)} \leq C\left\|\nabla \widetilde{z}_{\varepsilon}\right\|_{L^{2}\left(\Omega_{\varepsilon}\right)} \leq \frac{C}{\sqrt{\varepsilon}}
$$

$$
\left\|z_{\varepsilon}\right\|_{H^{1}\left(\Omega_{\varepsilon}\right)} \leq\left\|\delta_{\varepsilon}\right\|_{H(\Omega)}+\left\|\widetilde{z}_{\varepsilon}\right\|_{H^{1}\left(\Omega_{\varepsilon}\right)} \leq C\left[\left\|\nabla \delta_{\varepsilon}\right\|_{L^{2}(\Omega)}+\frac{1}{\sqrt{\varepsilon}}\right] \leq \frac{C}{\sqrt{\varepsilon}}
$$

Remark 4.3. We observe that the results of Lemma 4.2 and Theorem 4.1 hold for more general domains $\Omega_{\varepsilon}$, not necessarily perturbations of a rectangular domain $\Omega$.

As in Section 3 we define a semi-infinite strip $G_{g}$, which now has a non-flat boundary (see Fig. 2), by

$$
G_{g}=\left\{y=\left(y^{\prime}, y_{n}\right) \in Y^{\prime} \times \mathbb{R}: y_{n}<g\left(y^{\prime}\right)\right\} .
$$

The boundary of $G_{g}$ is divided in two disjoint parts $\partial G_{g}=\Gamma_{g} \cup \partial G_{\#}$, where $\partial G_{\#}=\partial G_{g} \backslash \Gamma_{g}$ and

$$
\Gamma_{g}=\left\{y=\left(y^{\prime}, y_{n}\right) \in Y^{\prime} \times \mathbb{R}: y_{n}=g\left(y^{\prime}\right)\right\} .
$$

Let $H_{\#, l o c}^{1}\left(G_{g}\right)$ be the space of functions in $G_{g}$ which are $Y^{\prime}$-periodic with respect to the $y^{\prime}$ variable and which belong to $H_{l o c}^{1}\left(G_{g}\right)$. Let $h(y)$ be the trace on $\Gamma_{g}$ of such a function in $H_{\#, l o c}^{1}\left(G_{g}\right)$. We consider the following problem

$$
\begin{cases}-\operatorname{div} A(y) \nabla \psi=0 & \text { on } G_{g}, \\ \psi=h & \text { on } \Gamma_{g}, \\ y^{\prime} \rightarrow \psi\left(y^{\prime}, y_{n}\right) & Y^{\prime} \text {-periodic. }\end{cases}
$$




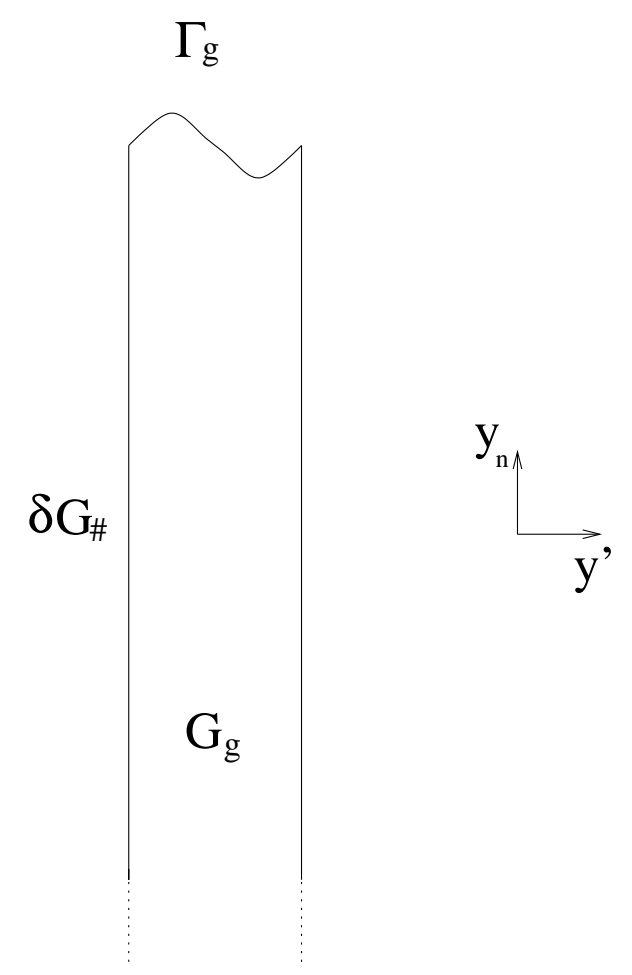

Figure 2. The semi-infinite strip $G_{g}$.

The existence, uniqueness, and behavior at infinity of solutions to problem (4.6) has been studied by many authors when the boundary $\Gamma_{g}$ is flat $\left(i . e . g\left(y^{\prime}\right)\right.$ is constant) $[11,14,15,22-26]$. The exponential decay of these solutions is often called a Phragmen-Lindelöf estimate or a Saint-Venant principle (in the context of elasticity). We propose another proof of these results which works also for a non-flat boundary $\Gamma_{g}$.

Lemma 4.4. There exists a unique solution $\psi$ of (4.6) in $H_{\#, l o c}^{1}\left(G_{g}\right)$. Moreover, there exist an exponent $\gamma>0$ and a unique real constant $d$ such that

$$
e^{-\gamma y_{n}}(\psi(y)-d) \in L^{2}\left(G_{g}\right), \quad e^{-\gamma y_{n}} \nabla \psi(y) \in L^{2}\left(G_{g}\right)^{n} .
$$

Remark 4.5. Clearly, the constant $d$ is the limit value at infinity of the solution $\psi$. We call $d$ a boundary layer tail. It is the only relevant information on the boundary layer $\psi$ away from the boundary $\Gamma_{g}$.

Proof. Let us define the space $D\left(G_{g}\right)=\left\{\phi \in H_{\#, l o c}^{1}\left(G_{g}\right):\|\nabla \phi\|_{L^{2}\left(G_{g}\right)^{n}}<+\infty\right\}$. The existence and uniqueness in $D\left(G_{g}\right)$ of the solution of (4.6) is clear: by the Lax-Milgram Lemma, there exists a unique solution $\psi$ of (4.6) which satisfies

$$
\|\nabla \psi\|_{L^{2}\left(G_{g}\right)^{n}} \leq C\|h\|_{H^{1 / 2}\left(\Gamma_{g}\right)}
$$

The difficulty is to prove that $\psi$ decays exponentially to a constant as stated in (4.7). When the extreme boundary $\Gamma_{g}$ is flat, it can be proved by means of Tartar Lemma (see, for instance, [24], Lem. 10.1 or [25], Th. 6, Cor. 2 and Prop. 4). However, this proof does not extend easily when $\Gamma_{g}$ is curved. We propose a new simpler proof in this context. Define the set $G_{k}=G_{g} \cap\left(Y^{\prime} \times(-\infty,-k)\right)$ and its boundary $\partial G_{k}=\Gamma_{k} \cup \partial G_{\#, k}$ 
where $\partial G_{\#, k}=\partial G_{k} \backslash \Gamma_{k}$ and $\Gamma_{k}=\left\{y \in G_{g}: y_{n}=-k\right\}$. For any $k \geq 1, \psi$ is also the solution in $G_{k}$ of

$$
\begin{cases}-\operatorname{div} A(y) \nabla \psi=0 & \text { on } G_{k} \\ \psi=\psi\left(y^{\prime},-k\right) & \text { on } \Gamma_{k}, \\ y^{\prime} \rightarrow \psi\left(y^{\prime}, y_{n}\right) & Y^{\prime} \text {-periodic. }\end{cases}
$$

Therefore, since the semi-infinite strips $G_{k}$ are all identical, up to a translation, estimate (4.8) holds in each $G_{k}$, i.e.

$$
\|\nabla \psi\|_{L^{2}\left(G_{k}\right)^{n}} \leq C\|\psi\|_{H^{1 / 2}\left(\Gamma_{k}\right)}
$$

where the constant $C$ is independent of $k$. Introducing $\psi_{k}(y)=\psi(y)-\int_{\Gamma_{k}} \psi d y^{\prime}$, it obviously satisfies

$$
\begin{cases}-\operatorname{div} A(y) \nabla \psi_{k}=0 & \text { on } G_{k} \\ \psi_{k}=\psi-\int_{\Gamma_{k}} \psi d y^{\prime} & \text { on } \Gamma_{k} \\ y^{\prime} \rightarrow \psi_{k}\left(y^{\prime}, y_{n}\right) & Y^{\prime} \text {-periodic. }\end{cases}
$$

Thus

$$
\|\nabla \psi\|_{L^{2}\left(G_{k}\right)^{n}}=\left\|\nabla \psi_{k}\right\|_{L^{2}\left(G_{k}\right)^{n}} \leq C\left\|\psi-\int_{\Gamma_{k}} \psi d y^{\prime}\right\|_{H^{1 / 2}\left(\Gamma_{k}\right)} .
$$

A Poincaré-Wirtinger type of inequality implies that

$$
\left\|\psi-\int_{\Gamma_{k}} \psi d y^{\prime}\right\|_{H^{1 / 2}\left(\Gamma_{k}\right)} \leq C\|\nabla \psi\|_{L^{2}\left(G_{k-1} \backslash G_{k}\right)^{n}}
$$

where, again, the constant $C$ does not depend on $k$. This implies that

$$
\|\nabla \psi\|_{L^{2}\left(G_{k}\right)^{n}}^{2} \leq C\left(\|\nabla \psi\|_{L^{2}\left(G_{k-1}\right)^{n}}^{2}-\|\nabla \psi\|_{L^{2}\left(G_{k}\right)^{n}}^{2}\right) .
$$

Thus, we deduce that

$$
\|\nabla \psi\|_{L^{2}\left(G_{k}\right)^{n}}^{2} \leq\left(\frac{C}{C+1}\right)^{k}\|\nabla \psi\|_{L^{2}\left(G_{0}\right)^{n}}^{2}
$$

Upon defining a positive constant $\gamma_{0}>0$ by

$$
\left(\frac{C}{C+1}\right)^{k}=\mathrm{e}^{-2 \gamma_{0} k}
$$

it is easily seen that (4.9) implies the first estimate in (4.7) for $\nabla \psi$ and any exponent $\gamma$ such that $0<\gamma<\gamma_{0}$. To obtain the other estimate on $\psi$ itself, we introduce a function $\bar{\psi}$ of the single variable $y_{n}$

$$
\bar{\psi}\left(y_{n}\right)=\int_{Y^{\prime} \times\left(y_{n}, y_{n}+1\right)} \psi(z) d z
$$

equivalently defined by

By virtue of (4.9) we get

$$
\bar{\psi}\left(y_{n}\right)=\int_{Y} \psi\left(z^{\prime}, z_{n}+y_{n}\right) d z
$$

$$
\left|\frac{d \bar{\psi}}{d y_{n}}\left(y_{n}\right)\right|=\left|\int_{Y} \frac{\partial \psi}{\partial y_{n}}\left(z^{\prime}, z_{n}+y_{n}\right) d z\right| \leq\|\nabla \psi\|_{L^{2}\left(Y^{\prime} \times\left(y_{n}, y_{n}+1\right)\right)^{n}} \leq C e^{\gamma_{0} y_{n}} .
$$


Therefore, there exists a constant $d$ such that

$$
\lim _{y_{n} \rightarrow-\infty} \bar{\psi}\left(y_{n}\right)=d
$$

and moreover

$$
\left|\bar{\psi}\left(y_{n}\right)-d\right| \leq C e^{\gamma_{0} y_{n}} .
$$

On the other hand, a slight modification of the Poincaré-Wirtinger inequality states that

$$
\left\|\psi(y)-\bar{\psi}\left(y_{n}\right)\right\|_{L^{2}\left(Y^{\prime} \times(-k,-k+1)\right)} \leq C\|\nabla \psi(y)\|_{L^{2}\left(Y^{\prime} \times(-k,-k+2)\right)^{n}},
$$

where the constant $C$ does not depend on $k$. Estimate (4.10) implies that

$$
\|\psi(y)-d\|_{L^{2}\left(Y^{\prime} \times(-k,-k+1)\right)} \leq\left\|\bar{\psi}\left(y_{n}\right)-d\right\|_{L^{2}\left(Y^{\prime} \times(-k,-k+1)\right)}+C\|\nabla \psi(y)\|_{L^{2}\left(Y^{\prime} \times(-k,-k+2)\right)^{n}} \leq C e^{-\gamma_{0} k},
$$

which immediately leads to the desired result upon summing over $k$ for any $0<\gamma<\gamma_{0}$.

Let $\psi$ be the solution of the problem (4.6). By translation and rescaling, we define

$$
\psi_{\varepsilon}\left(x^{\prime}, x_{n}\right)=\psi\left(\frac{x^{\prime}}{\varepsilon}, \frac{x_{n}-(1-\varepsilon)}{\varepsilon}\right)-d,
$$

where $d$ is the constant limit defined in Lemma 4.4. Clearly, $\psi_{\varepsilon}$ belongs to $H^{1}\left(\Omega_{\varepsilon}\right)$ and satisfies

$$
\begin{cases}-\operatorname{div} A_{\varepsilon} \nabla \psi_{\varepsilon}=0 & \text { in } \Omega_{\varepsilon}, \\ \psi_{\varepsilon}\left(x^{\prime}, x_{n}\right)=\psi\left(\frac{x^{\prime}}{\varepsilon}, \frac{g_{\varepsilon}\left(x^{\prime}\right)-1+\varepsilon}{\varepsilon}\right)-d=h\left(\frac{x^{\prime}}{\varepsilon}\right)-d & \text { on } \Gamma_{\varepsilon}, \\ x^{\prime} \rightarrow \psi_{\varepsilon}\left(x^{\prime}, x_{n}\right) & \varepsilon Y^{\prime} \text {-periodic. }\end{cases}
$$

A simple computation shows that

$$
\left\|\psi_{\varepsilon}(x) \exp \left[-\gamma\left(\frac{x_{n}-g_{\varepsilon}\left(x^{\prime}\right)}{\varepsilon}\right)\right]\right\|_{L^{2}\left(\Omega_{\varepsilon}\right)}^{2}=\varepsilon\left\|(\psi(y)-d) \exp \left[-\gamma\left(y_{n}-g\left(y^{\prime}\right)\right)\right]\right\|_{L^{2}\left(G_{g}\right)}^{2}+r_{\varepsilon},
$$

where $r_{\varepsilon}$ is a negative remaider of order $\varepsilon \exp (-C / \varepsilon)$ with $C>0$. Therefore, by Lemma 4.4, the sequence $\frac{1}{\sqrt{\varepsilon}} \psi_{\varepsilon} \exp \left[-\gamma\left(\frac{x_{n}-g_{\varepsilon}\left(x^{\prime}\right)}{\varepsilon}\right)\right]$ is uniformly bounded in $L^{2}\left(\Omega_{\varepsilon}\right)$. A similar argument implies that the sequence $\sqrt{\varepsilon}\left(\nabla \psi_{\varepsilon}\right) \exp \left[-\gamma\left(\frac{x_{n}-g_{\varepsilon}\left(x^{\prime}\right)}{\varepsilon}\right)\right]$ is also uniformly bounded in $L^{2}\left(\Omega_{\varepsilon}\right)^{n}$. In other words

$$
\left\|\psi_{\varepsilon} \exp \left[-\gamma\left(\frac{x_{n}-g_{\varepsilon}\left(x^{\prime}\right)}{\varepsilon}\right)\right]\right\|_{L^{2}\left(\Omega_{\varepsilon}\right)} \leq C \sqrt{\varepsilon} \quad \text { and } \quad\left\|\nabla \psi_{\varepsilon} \exp \left[-\gamma\left(\frac{x_{n}-g_{\varepsilon}\left(x^{\prime}\right)}{\varepsilon}\right)\right]\right\|_{L^{2}\left(\Omega_{\varepsilon}\right)^{n}} \leq \frac{C}{\sqrt{\varepsilon}} \text {. }
$$

Finally, we define

$$
\widetilde{\psi}_{\varepsilon}\left(x^{\prime}, x_{n}\right)=\psi_{\varepsilon}\left(x^{\prime}, x_{n}\right) V_{\varepsilon}\left(x^{\prime}, x_{n}\right),
$$

where $V_{\varepsilon}: \bar{\Omega}_{\varepsilon} \rightarrow \mathbb{R}$ is a sequence of functions which is bounded in $W^{1, \infty}\left(\Omega_{\varepsilon}\right)$, i.e. $\left\|V_{\varepsilon}\right\|_{W^{1, \infty}\left(\Omega_{\varepsilon}\right)} \leq C$, such that $V_{\varepsilon}=0$ on $\Gamma_{0}$, and $x^{\prime} \rightarrow V_{\varepsilon}\left(x^{\prime}, x_{n}\right)$ is periodic. There are many possible choices of such sequences: for instance, we may take $V_{\varepsilon}\left(x^{\prime}, x_{n}\right)=V\left(x^{\prime}\right) \frac{x_{n}}{g_{\varepsilon}\left(x^{\prime}\right)}$ where $V$ is a smooth periodic function. 
We now state a "periodic" version of Lemma 3.2 and Corollary 3.3. We assume in the sequel that $h$ is the trace on $Y^{\prime}$ of a function in $W_{\#}^{1, \infty}(Y)$.

Lemma 4.6. Let $\widetilde{\psi}_{\varepsilon}$ be defined by (4.12). Let $w_{\varepsilon}$ be the unique solution in $H^{1}\left(\Omega_{\varepsilon}\right)$ of

$$
\begin{cases}-\operatorname{div} A_{\varepsilon} \nabla w_{\varepsilon}=0 & \text { in } \Omega_{\varepsilon}, \\ w_{\varepsilon}(x)=V_{\varepsilon}(x)\left(h\left(\frac{x^{\prime}}{\varepsilon}\right)-d\right) & \text { on } \Gamma_{\varepsilon} \cup \Gamma_{0}, \\ x^{\prime} \rightarrow w_{\varepsilon}\left(x^{\prime}, x_{n}\right) & \text { periodic on } \Gamma_{\#} .\end{cases}
$$

Then $\widetilde{\psi}_{\varepsilon}=w_{\varepsilon}$ on $\Gamma_{\varepsilon} \cup \Gamma_{0}$ and

$$
\left\|w_{\varepsilon}-\widetilde{\psi}_{\varepsilon}\right\|_{H\left(\Omega_{\varepsilon}\right)} \leq C \sqrt{\varepsilon} .
$$

Proof. By construction we have $\widetilde{\psi}_{\varepsilon}=w_{\varepsilon}$ on $\Gamma_{\varepsilon} \cup \Gamma_{0}$ and $\widetilde{\psi}_{\varepsilon}-w_{\varepsilon} \in H\left(\Omega_{\varepsilon}\right)$. Let us prove that

$$
\left\|-\operatorname{div} A_{\varepsilon} \nabla\left(w_{\varepsilon}-\widetilde{\psi}_{\varepsilon}\right)\right\|_{H^{\prime}\left(\Omega_{\varepsilon}\right)} \leq C \sqrt{\varepsilon}
$$

Let $\phi \in H\left(\Omega_{\varepsilon}\right)$ be a test function; then

$$
\begin{aligned}
\int_{\Omega_{\varepsilon}}-\operatorname{div}\left[A_{\varepsilon} \nabla\left(w_{\varepsilon}-\widetilde{\psi}_{\varepsilon}\right)\right] \phi d x^{\prime} d x_{n} & =\int_{\Omega_{\varepsilon}} \operatorname{div} A_{\varepsilon} \nabla \widetilde{\psi}_{\varepsilon} \phi d x^{\prime} d x_{n} \\
& =\int_{\Omega_{\varepsilon}}\left\{\operatorname{div}\left[A_{\varepsilon} \nabla V_{\varepsilon}\right] \psi_{\varepsilon}+A_{\varepsilon} \nabla V_{\varepsilon} \nabla \psi_{\varepsilon}+A_{\varepsilon} \nabla \psi_{\varepsilon} \nabla V_{\varepsilon}\right\} \phi d x^{\prime} d x_{n} \\
& =-\int_{\Omega_{\varepsilon}}\left[A_{\varepsilon} \nabla V_{\varepsilon} \nabla\left(\psi_{\varepsilon} \phi\right)-A_{\varepsilon} \nabla V_{\varepsilon} \nabla \psi_{\varepsilon} \phi-A_{\varepsilon} \nabla \psi_{\varepsilon} \nabla V_{\varepsilon} \phi\right] d x^{\prime} d x_{n} \\
& =\int_{\Omega_{\varepsilon}}\left[-A_{\varepsilon} \nabla V_{\varepsilon} \nabla \phi \psi_{\varepsilon}+A_{\varepsilon} \nabla \psi_{\varepsilon} \nabla V_{\varepsilon} \phi\right] d x^{\prime} d x_{n} \\
& \leq C \int_{\Omega_{\varepsilon}}\left[\left|\psi_{\varepsilon}\right||\nabla \phi|+\left|\nabla \psi_{\varepsilon}\right||\phi|\right] d x^{\prime} d x_{n} \\
& =C\left[I_{1}+I_{2}\right] .
\end{aligned}
$$

Let us study $I_{1}$ and $I_{2}$. Clearly

By (4.11), it follows

$$
I_{1} \leq\left\|\psi_{\varepsilon}\right\|_{L^{2}\left(\Omega_{\varepsilon}\right)}\|\phi\|_{H\left(\Omega_{\varepsilon}\right)}
$$

$$
\left\|\psi_{\varepsilon}\right\|_{L^{2}\left(\Omega_{\varepsilon}\right)} \leq\left\|\psi_{\varepsilon} \exp \left[-\gamma\left(\frac{x_{n}-g_{\varepsilon}\left(x^{\prime}\right)}{\varepsilon}\right)\right]\right\|_{L^{2}\left(\Omega_{\varepsilon}\right)} \leq C \sqrt{\varepsilon}
$$

which implies

$$
I_{1} \leq C \sqrt{\varepsilon}\|\phi\|_{H\left(\Omega_{\varepsilon}\right)}
$$

In order to control $I_{2}$, we divide the domain $\Omega_{\varepsilon}$ in strips $C_{\varepsilon}^{k}$ such that $\Omega_{\varepsilon}=\cup_{k=1}^{\varepsilon^{-1}} C_{\varepsilon}^{k}$ defined by

$$
C_{\varepsilon}^{k}=\left\{\left(x^{\prime}, x_{n}\right) \in \Omega_{\varepsilon}: 1-(k-1) \varepsilon \geq x_{n} \geq 1-k \varepsilon\right\} .
$$

Introducing $D_{\varepsilon}^{k}=\cup_{l=1}^{k} C_{\varepsilon}^{l}$, $\phi$ (which vanishes on $\Gamma_{\varepsilon}$ ) satisfies the following Poincaré inequality

$$
\|\phi\|_{L^{2}\left(D_{\varepsilon}^{k}\right)} \leq C k \varepsilon\|\nabla \phi\|_{L^{2}\left(D_{\varepsilon}^{k}\right)^{n}}
$$


where the constant $C>0$ does not depend on $k$ and $\varepsilon$. Then

$$
\begin{aligned}
I_{2}= & \sum_{k=1}^{\varepsilon^{-1}} \int_{C_{\varepsilon}^{k}}\left|\nabla \psi_{\varepsilon}\right||\phi| d x^{\prime} d x_{n} \\
& \leq \sum_{k=1}^{\varepsilon^{-1}}\left\|\nabla \psi_{\varepsilon}\right\|_{L^{2}\left(C_{\varepsilon}^{k}\right)}\|\phi\|_{L^{2}\left(D_{\varepsilon}^{k}\right)} \\
& \leq C \sum_{k=1}^{\varepsilon^{-1}} \frac{\sqrt{\varepsilon}}{\varepsilon} \exp \left[-\gamma_{0} k\right] k \varepsilon\|\nabla \phi\|_{L^{2}\left(\Omega_{\varepsilon}\right)^{n}} \\
& \leq C \sqrt{\varepsilon}\|\nabla \phi\|_{L^{2}\left(\Omega_{\varepsilon}\right)^{n}} \sum_{k=1}^{\varepsilon^{-1}} k \exp \left[-\gamma_{0} k\right] \leq C \sqrt{\varepsilon}\|\phi\|_{H\left(\Omega_{\varepsilon}\right)},
\end{aligned}
$$

where we used (4.9) to bound $\psi_{\varepsilon}$. Taking into account (4.16) and (4.15), it follows

$$
I_{1}+I_{2} \leq C \sqrt{\varepsilon}\|\phi\|_{H\left(\Omega_{\varepsilon}\right)} .
$$

Passing to the supremum with respect to $\phi \in H\left(\Omega_{\varepsilon}\right)$ with $\|\phi\|_{H\left(\Omega_{\varepsilon}\right)}=1$, we obtain

$$
\left\|-\operatorname{div} A_{\varepsilon} \nabla\left(w_{\varepsilon}-\widetilde{\psi}_{\varepsilon}\right)\right\|_{H^{\prime}\left(\Omega_{\varepsilon}\right)}=\sup _{\substack{\phi \in H\left(\Omega_{\varepsilon}\right) \\\|\phi\|_{H\left(\Omega_{\varepsilon}\right)}}} \int_{\Omega_{\varepsilon}}-\operatorname{div}\left[A_{\varepsilon} \nabla\left(w_{\varepsilon}-\widetilde{\psi}_{\varepsilon}\right)\right] \phi d x^{\prime} d x_{n} \leq C \sqrt{\varepsilon}
$$

which is nothing but (4.13). Eventually, energy estimates yield

$$
\begin{aligned}
\left\|w_{\varepsilon}-\widetilde{\psi}_{\varepsilon}\right\|_{H\left(\Omega_{\varepsilon}\right)}^{2} & \leq \frac{1}{\lambda} \int_{\Omega_{\varepsilon}} A_{\varepsilon} \nabla\left(w_{\varepsilon}-\widetilde{\psi}_{\varepsilon}\right) \nabla\left(w_{\varepsilon}-\widetilde{\psi}_{\varepsilon}\right) d x^{\prime} d x_{n} \leq \frac{1}{\lambda} \int_{\Omega_{\varepsilon}}-\operatorname{div}\left[A_{\varepsilon} \nabla\left(w_{\varepsilon}-\widetilde{\psi}_{\varepsilon}\right)\right]\left(w_{\varepsilon}-\widetilde{\psi}_{\varepsilon}\right) d x^{\prime} d x_{n} \\
& \leq \frac{1}{\lambda}\left\|-\operatorname{div} A_{\varepsilon} \nabla\left(w_{\varepsilon}-\widetilde{\psi}_{\varepsilon}\right)\right\|_{H^{\prime}\left(\Omega_{\varepsilon}\right)}\left\|w_{\varepsilon}-\widetilde{\psi}_{\varepsilon}\right\|_{H\left(\Omega_{\varepsilon}\right)} \leq C \sqrt{\varepsilon}\left\|w_{\varepsilon}-\widetilde{\psi}_{\varepsilon}\right\|_{H\left(\Omega_{\varepsilon}\right)} .
\end{aligned}
$$

The thesis is then accomplished.

As a consequence of Lemma 4.6, we obtain the following estimate for $w_{\varepsilon}$.

Corollary 4.7. Let $w_{\varepsilon}$ be defined as in Lemma 4.6. Then

$$
\begin{aligned}
\left\|w_{\varepsilon}\right\|_{H^{1}\left(\Omega_{\varepsilon}\right)} \leq \frac{C}{\sqrt{\varepsilon}} \\
\left\|w_{\varepsilon}\right\|_{L^{2}\left(\Omega_{\varepsilon}\right)} \leq C \sqrt{\varepsilon},
\end{aligned}
$$

and, for every open set $\omega \subset \subset \Omega$, there exists a constant $C>0$ such that

$$
\left\|w_{\varepsilon}\right\|_{H^{1}(\omega)} \leq C \sqrt{\varepsilon}
$$

Remark 4.8. We observe that Lemma 4.6 is more precise than this corollary, but $(4.17,4.18)$ and $(4.19)$ are just the correct estimates that we require in the sequel. We conjecture that they hold for every oscillating domain, and that their proof could be obtained directly without using Lemma 4.6. 
Proof. Estimate (4.17) is an immediate consequence of Lemma 4.2 since

$$
\left\|V_{\varepsilon}(x)\left[h\left(\frac{x^{\prime}}{\varepsilon}\right)-d\right]\right\|_{L^{\infty}\left(\Omega_{\varepsilon}\right)} \leq C \quad \text { and } \quad\left\|\nabla\left(V_{\varepsilon}(x)\left[h\left(\frac{x^{\prime}}{\varepsilon}\right)-d\right]\right)\right\|_{L^{\infty}\left(\Omega_{\varepsilon}\right)^{n}} \leq \frac{C}{\varepsilon} .
$$

In order to obtain (4.18), we observe that, by Lemma 4.6, it follows that

$$
\left\|w_{\varepsilon}\right\|_{L^{2}\left(\Omega_{\varepsilon}\right)} \leq\left\|w_{\varepsilon}-\widetilde{\psi}_{\varepsilon}\right\|_{L^{2}\left(\Omega_{\varepsilon}\right)}+\left\|\widetilde{\psi}_{\varepsilon}\right\|_{L^{2}\left(\Omega_{\varepsilon}\right)} \leq\left\|w_{\varepsilon}-\widetilde{\psi}_{\varepsilon}\right\|_{H\left(\Omega_{\varepsilon}\right)}+\left\|\widetilde{\psi}_{\varepsilon}\right\|_{L^{2}\left(\Omega_{\varepsilon}\right)} \leq C \sqrt{\varepsilon}+\left\|\widetilde{\psi}_{\varepsilon}\right\|_{L^{2}\left(\Omega_{\varepsilon}\right)} .
$$

Moreover, by (4.12) and (4.14), it follows

$$
\left\|\widetilde{\psi}_{\varepsilon}\right\|_{L^{2}\left(\Omega_{\varepsilon}\right)} \leq C\left\|\psi_{\varepsilon}\right\|_{L^{2}\left(\Omega_{\varepsilon}\right)} \leq C \sqrt{\varepsilon} .
$$

Finally, for proving (4.19), we choose an open set $\omega$ such that $\omega \subset \subset \Omega$. Let $\omega^{\prime}$ be another open set such that $\omega \subset \subset \omega^{\prime} \subset \subset \Omega$. Let us choose $\varepsilon>0$ sufficiently small such that $\omega^{\prime} \subset \subset \Omega_{\varepsilon} \subset \Omega$. Let $\widetilde{\psi}_{\varepsilon}$ be defined by (4.12). By Lemma 4.6, it follows that

$$
\left\|w_{\varepsilon}\right\|_{H^{1}(\omega)} \leq\left\|w_{\varepsilon}-\widetilde{\psi}_{\varepsilon}\right\|_{H\left(\Omega_{\varepsilon}\right)}+\left\|\widetilde{\psi}_{\varepsilon}\right\|_{H^{1}(\omega)} \leq C \sqrt{\varepsilon}+\left\|\widetilde{\psi}_{\varepsilon}\right\|_{H^{1}(\omega)} .
$$

Hence, in order to obtain the desired inequality it is enough to give a proper bound for $\left\|\widetilde{\psi}_{\varepsilon}\right\|_{H^{1}(\omega)}$. Introducing $k^{2}=\inf d\left(\omega, \partial \omega^{\prime}\right)>0,(4.11)$ and (4.12) imply that

$$
\begin{aligned}
\left\|\widetilde{\psi}_{\varepsilon}\right\|_{H^{1}(\omega)} & \leq C\left\|\psi_{\varepsilon}\right\|_{H^{1}(\omega)} \leq C \exp \left(-\gamma \frac{k^{2}}{\varepsilon}\right)\left[\int_{\omega}\left(\exp \left(-2 \gamma\left(\frac{x_{n}-g_{\varepsilon}\left(x^{\prime}\right)}{\varepsilon}\right)\right)\left(\left|\psi_{\varepsilon}\right|^{2}+\left|\nabla \psi_{\varepsilon}\right|^{2}\right)\right) d x^{\prime} d x_{n}\right]^{\frac{1}{2}} \\
& \leq C \frac{1}{\sqrt{\varepsilon}} \exp \left(-\gamma \frac{k^{2}}{\varepsilon}\right) \leq C \sqrt{\varepsilon}
\end{aligned}
$$

which is the desired result.

\section{The CaSe of Laplace EQUation}

This section is the sequel of Section 4 in the particular case where the coefficients are constant, i.e. there is no interaction between homogenization and the oscillating boundary. We still assume that $\Omega=(0,1)^{n}$. For simplicity we assume that $a_{i j}(y)=\delta_{i j}$, i.e. equation (4.1) reduces to Laplace equation

$$
\begin{cases}-\Delta u_{\varepsilon}=f & \text { in } \Omega_{\varepsilon}, \\ u_{\varepsilon}=0 & \text { on } \Gamma_{\varepsilon} \cup \Gamma_{0}, \\ x^{\prime} \rightarrow u_{\varepsilon}\left(x^{\prime}, x_{n}\right) & \text { periodic on } \Gamma_{\#},\end{cases}
$$

and the homogenized equation (4.4) is simply

$$
\begin{cases}-\Delta u=f & \text { in } \Omega, \\ u=0 & \text { on } \Gamma \cup \Gamma_{0}, \\ x^{\prime} \rightarrow u\left(x^{\prime}, x_{n}\right) & \text { periodic on } \Gamma_{\#} .\end{cases}
$$

This type of problem has often been addressed in the past (see e.g. [16-18,21,27]). Specifically, a similar case was treated in $[1,2,20]$, in the context of homogenization of a periodic coating for an obstacle reflecting an electromagnetic wave, and in $[3,4]$, in connection with the study of wall laws for fluid flows over a rough boundary. All the results in this section are merely a unified rephrasing of these previous works. 
With these further assumptions Theorem 4.1 can easily be improved (see [21] for a proof of Lem. 5.1 and Th. 5.2).

Lemma 5.1. Let $u_{\varepsilon}$ be the unique solution in $H\left(\Omega_{\varepsilon}\right)$ of (5.1) and $u$ the unique solution in $H(\Omega)$ of (5.2). Assume that $u \in W^{2, \infty}(\Omega)$. Then

$$
\left\|u_{\varepsilon}(x)-u(x)\right\|_{H^{1}\left(\Omega_{\varepsilon}\right)} \leq C \sqrt{\varepsilon} .
$$

We define a boundary layer term $\vartheta_{\varepsilon}$ as the unique solution in $H^{1}\left(\Omega_{\varepsilon}\right)$ of

$$
\begin{cases}-\Delta \vartheta_{\varepsilon}=0 & \text { in } \Omega_{\varepsilon}, \\ \vartheta_{\varepsilon}=\left[1-g\left(\frac{x^{\prime}}{\varepsilon}\right)\right] \frac{\partial u}{\partial x_{n}}\left(x^{\prime}, 1\right) & \text { on } \Gamma_{\varepsilon}, \\ \vartheta_{\varepsilon}=0 & \text { on } \Gamma_{0}, \\ x^{\prime} \rightarrow \vartheta_{\varepsilon}\left(x^{\prime}, x_{n}\right) & \text { periodic on } \Gamma_{\#} .\end{cases}
$$

Theorem 5.2. Let $u_{\varepsilon}$ be the unique solution in $H\left(\Omega_{\varepsilon}\right)$ of (5.1) and $u$ the unique solution in $H(\Omega)$ of (5.2). Assume that $u \in W^{3, \infty}(\Omega)$. Then,

$$
\left\|u_{\varepsilon}(x)-u(x)-\varepsilon \vartheta_{\varepsilon}(x)\right\|_{H^{1}\left(\Omega_{\varepsilon}\right)} \leq C \varepsilon^{\frac{3}{2}} .
$$

Theorem 5.2 is not entirely satisfactory since the boundary layer $\vartheta_{\varepsilon}$ is not easy to compute in the oscillating domain $\Omega_{\varepsilon}$. In order to improve Theorem 5.2, we want to replace $\vartheta_{\varepsilon}$ by a function computed in the fixed domain $\Omega$ and still obtain an estimate similar to (5.4), at least away from the boundary $\Gamma_{\varepsilon}$. For this purpose, we introduce the boundary layer tail $d$ defined in Lemma 4.4 for the boundary data $h(y)=1-g(y)($ recall that $d$ is the constant limit value at infinity of the solution $\psi$ of $(4.6))$. Let $\phi$ be the unique solution in $H^{1}(\Omega)$ of

$$
\begin{cases}-\Delta \phi=0 & \text { in } \Omega, \\ \phi=d \frac{\partial u}{\partial x_{n}}\left(x^{\prime}, 1\right) & \text { on } \Gamma \\ \phi=0 & \text { on } \Gamma_{0}, \\ x^{\prime} \rightarrow \phi\left(x^{\prime}, x_{n}\right) & \text { periodic on } \Gamma_{\#} .\end{cases}
$$

Theorem 5.3. Let $u_{\varepsilon}$ be the unique solution in $H\left(\Omega_{\varepsilon}\right)$ of (5.1) and $u$ the unique solution in $H(\Omega)$ of (5.2). Assume that $u \in W^{3, \infty}(\Omega)$. Assume that the solution $\phi$ of $(5.5)$ belongs to $W^{2, \infty}(\Omega)$. Then, for any open set $\omega \subset \subset \Omega$, there exists a constant $C>0$ such that

$$
\left\|u_{\varepsilon}(x)-u(x)-\varepsilon \phi(x)\right\|_{H^{1}(\omega)} \leq C \varepsilon^{\frac{3}{2}}
$$

Proof. Let $\omega \subset \subset \Omega$. Let $\varepsilon>0$ be small enough in such a way that $\omega \subset \subset \Omega_{\varepsilon} \subset \Omega$. Let $\vartheta_{\varepsilon}$ be the solution of (5.3). Then

$$
\left\|u_{\varepsilon}-u-\varepsilon \phi\right\|_{H^{1}(\omega)} \leq\left\|u_{\varepsilon}-u-\varepsilon \vartheta_{\varepsilon}\right\|_{H^{1}\left(\Omega_{\varepsilon}\right)}+\varepsilon\left\|\left(\vartheta_{\varepsilon}-\phi\right)\right\|_{H^{1}(\omega)} \leq C \varepsilon^{\frac{3}{2}}+\varepsilon\left\|\vartheta_{\varepsilon}-\phi\right\|_{H^{1}(\omega)},
$$

where the last inequality follows from (5.4). Hence, to conclude the proof, it suffices to give a proper bound for $\left\|\vartheta_{\varepsilon}-\phi\right\|_{H^{1}(\omega)}$. Remark that $\vartheta_{\varepsilon}-\phi$ satisfies

$$
\begin{cases}-\Delta\left(\vartheta_{\varepsilon}-\phi\right)=0 & \text { in } \Omega_{\varepsilon}, \\ \vartheta_{\varepsilon}-\phi=\left[1-g\left(\frac{x^{\prime}}{\varepsilon}\right)\right] \frac{\partial u}{\partial x_{n}}\left(x^{\prime}, 1\right)-\phi(x) & \text { on } \Gamma_{\varepsilon}, \\ \vartheta_{\varepsilon}-\phi=0 & \text { on } \Gamma_{0}, \\ x^{\prime} \rightarrow \vartheta_{\varepsilon}\left(x^{\prime}, x_{n}\right)-\phi\left(x^{\prime}, x_{n}\right) & \text { periodic on } \Gamma_{\#},\end{cases}
$$


where, by a Taylor expansion on $\Gamma_{\varepsilon}$,

$$
\phi(x)=d \frac{\partial u}{\partial x_{n}}\left(x^{\prime}, 1\right)+\int_{1}^{g_{\varepsilon}\left(x^{\prime}\right)} \frac{\partial \phi}{\partial x_{n}}\left(x^{\prime}, \tau\right) d \tau
$$

Hence,

$$
\vartheta_{\varepsilon}-\phi=\left[1-g\left(\frac{x^{\prime}}{\varepsilon}\right)-d\right] \frac{\partial u}{\partial x_{n}}\left(x^{\prime}, 1\right)-\int_{1}^{g_{\varepsilon}\left(x^{\prime}\right)} \frac{\partial \phi}{\partial x_{n}}\left(x^{\prime}, \tau\right) d \tau \quad \text { on } \Gamma_{\varepsilon} .
$$

Set $\vartheta_{\varepsilon}-\phi=w_{\varepsilon}+v_{\varepsilon}$ where $w_{\varepsilon}$ satisfies

$$
\begin{cases}-\Delta w_{\varepsilon}=0 & \text { in } \Omega_{\varepsilon}, \\ w_{\varepsilon}=\left[1-g\left(\frac{x^{\prime}}{\varepsilon}\right)-d\right] \frac{\partial u}{\partial x_{n}}\left(x^{\prime}, 1\right) & \text { on } \Gamma_{\varepsilon}, \\ w_{\varepsilon}=0 & \text { on } \Gamma_{0}, \\ x^{\prime} \rightarrow w_{\varepsilon}\left(x^{\prime}, x_{n}\right) & \text { periodic on } \Gamma_{\#},\end{cases}
$$

and $v_{\varepsilon}$ satisfies

$$
\begin{cases}-\Delta v_{\varepsilon}=0 & \text { in } \Omega_{\varepsilon}, \\ v_{\varepsilon}=\varepsilon \int_{0}^{1-g\left(\frac{x^{\prime}}{\varepsilon}\right)} \frac{\partial \phi}{\partial x_{n}}\left(x^{\prime}, 1-\varepsilon \tau\right) d \tau=\bar{\phi}_{\varepsilon} & \text { on } \Gamma_{\varepsilon}, \\ v_{\varepsilon}=0 & \text { on } \Gamma_{0}, \\ x^{\prime} \rightarrow v_{\varepsilon}\left(x^{\prime}, x_{n}\right) & \text { periodic on } \Gamma_{\#} .\end{cases}
$$

By Lemma 4.6 and Corollary 4.7 with $V_{\varepsilon}\left(x^{\prime}, x_{n}\right)=\frac{\partial u}{\partial x_{n}}\left(x^{\prime}, 1\right) \frac{x_{n}}{g_{\varepsilon}\left(x^{\prime}\right)}$ and $h\left(y^{\prime}\right)=1-g\left(y^{\prime}\right)$, it follows that $\left\|w_{\varepsilon}\right\|_{H^{1}(\omega)} \leq C \sqrt{\varepsilon}$. Moreover, since $\bar{\phi}_{\varepsilon}$ satisfies the estimates

$$
\left\|\bar{\phi}_{\varepsilon}\right\|_{L^{\infty}\left(\Omega_{\varepsilon}\right)} \leq C \varepsilon \quad \text { and } \quad\left\|\nabla \bar{\phi}_{\varepsilon}\right\|_{L^{\infty}\left(\Omega_{\varepsilon}\right)^{n}} \leq C,
$$

by Lemma 4.2 , we obtain that $\left\|v_{\varepsilon}\right\|_{H^{1}\left(\Omega_{\varepsilon}\right)} \leq C \varepsilon^{\frac{1}{2}}$. This yields the desired result $\left\|\vartheta_{\varepsilon}-\phi\right\|_{H^{1}(\omega)} \leq C \sqrt{\varepsilon}$.

Remark 5.4. In the spirit of Remark 3.11 , we emphasize that it is not necessary to do a separate computation for $\phi$. Indeed, defining $\bar{u}_{\varepsilon}$ as the unique solution in $H^{1}(\Omega)$ of

$$
\begin{cases}-\Delta \bar{u}_{\varepsilon}=f & \text { in } \Omega, \\ \bar{u}_{\varepsilon}=\varepsilon d \frac{\partial \bar{u}_{\varepsilon}}{\partial x_{n}} & \text { on } \Gamma, \\ \bar{u}_{\varepsilon}=0 & \text { on } \Gamma_{0} \\ x^{\prime} \rightarrow \bar{u}_{\varepsilon}\left(x^{\prime}, x_{n}\right) & \text { periodic on } \Gamma_{\#},\end{cases}
$$

it is easily seen that $\bar{u}_{\varepsilon}$ differs from $u+\varepsilon \phi$ by a term of order $\varepsilon^{2}$ in the $H^{1}(\Omega)$-norm. The existence of a solution of (5.6) is always guaranteed for small $\varepsilon$, whatever the sign of the constant $d$ is. Remark that $\bar{u}_{\varepsilon}$ depends on $\varepsilon$ but is not an oscillating function. Therefore, we have

$$
\left\|u_{\varepsilon}(x)-\bar{u}_{\varepsilon}(x)\right\|_{H^{1}(\omega)} \leq C \varepsilon^{\frac{3}{2}} .
$$




\section{The General CASE}

In this section we come back to the general setting of Section 4 , and still assume that $\Omega=(0,1)^{n}$. We study the boundary layers $u_{i}^{b l, \varepsilon}$ defined in (4.3). We begin with the zero-th order term $u_{0}^{b l, \varepsilon}$ which is defined by

$$
\begin{cases}-\operatorname{div} A_{\varepsilon} \nabla u_{0}^{b l, \varepsilon}=0 & \text { in } \Omega_{\varepsilon} \\ u_{0}^{b l, \varepsilon}=-u & \text { on } \Gamma_{\varepsilon} \cup \Gamma_{0}, \\ x^{\prime} \rightarrow u_{0}^{b l, \varepsilon}\left(x^{\prime}, x_{n}\right) & \text { periodic on } \Gamma_{\#} .\end{cases}
$$

We decompose it in the sum of three terms $u_{0}^{b l, \varepsilon}(x)=\varepsilon\left(u_{0}^{b l}(x)+v_{0}^{b l, \varepsilon}(x)+w_{0}^{b l, \varepsilon}(x)\right)$ where $u_{0}^{b l}$ satisfies

$$
\begin{cases}-\operatorname{div} A^{*} \nabla u_{0}^{b l}=0 & \text { in } \Omega, \\ u_{0}^{b l}=d \frac{\partial u}{\partial x_{n}} & \text { on } \Gamma, \\ u_{0}^{b l}=0 & \text { on } \Gamma_{0}, \\ x^{\prime} \rightarrow u_{0}^{b l}\left(x^{\prime}, x_{n}\right) & \text { periodic on } \Gamma_{\#},\end{cases}
$$

$v_{0}^{b l, \varepsilon}$ is the unique solution of

$$
\begin{cases}-\operatorname{div} A_{\varepsilon} \nabla v_{0}^{b l, \varepsilon}=\operatorname{div} A_{\varepsilon} \nabla u_{0}^{b l} & \text { in } \Omega_{\varepsilon} \\ v_{0}^{b l, \varepsilon}=0 & \text { on } \Gamma_{\varepsilon} \cup \Gamma_{0} \\ x^{\prime} \rightarrow v_{0}^{b l, \varepsilon}\left(x^{\prime}, x_{n}\right) & \text { periodic on } \Gamma_{\#}\end{cases}
$$

and $w_{0}^{b l, \varepsilon}$ is the unique solution of

$$
\begin{cases}-\operatorname{div} A_{\varepsilon} \nabla w_{0}^{b l, \varepsilon}=0 & \text { in } \Omega_{\varepsilon}, \\ w_{0}^{b l, \varepsilon}=-\frac{u}{\varepsilon}-u_{0}^{b l} & \text { on } \Gamma_{\varepsilon} \\ w_{0}^{b l, \varepsilon}=0 & \text { on } \Gamma_{0}, \\ x^{\prime} \rightarrow w_{0}^{b l, \varepsilon}\left(x^{\prime}, x_{n}\right) & \text { periodic on } \Gamma_{\#} \cdot\end{cases}
$$

In (6.2) $d$ is the constant (or boundary layer tail) defined by Lemma 4.4 applied to $h(y)=1-g(y)$. Assuming that $u \in W^{2, \infty}(\Omega), u_{0}^{b l}(x)$ does not depend on $\varepsilon$ and satisfies the a priori estimate

$$
\left\|u_{0}^{b l}\right\|_{H^{1}(\Omega)} \leq C
$$

On the other hand, $v_{0}^{b l, \varepsilon}$ is not a boundary layer and classical a priori estimates in homogenization yield

$$
\left\|v_{0}^{b l, \varepsilon}\right\|_{H\left(\Omega_{\varepsilon}\right)} \leq C .
$$

Finally, the following result provides uniform estimates for $w_{0}^{b l, \varepsilon}$.

Lemma 6.1. Assume that $u \in W^{3, \infty}(\Omega)$. Then, the solution $w_{0}^{b l, \varepsilon}$ of (6.4) satisfies

$$
\left\|w_{0}^{b l, \varepsilon}\right\|_{H^{1}\left(\Omega_{\varepsilon}\right)} \leq \frac{C}{\sqrt{\varepsilon}}
$$

and, for every open set $\omega \subset \subset \Omega$, there exists a positive constant $C$ such that

$$
\left\|w_{0}^{b l, \varepsilon}\right\|_{H^{1}(\omega)} \leq C \sqrt{\varepsilon}
$$


Proof. By a Taylor expansion of $u$ and $u_{0}^{b l}$ with integral rest, we may write

$$
w_{0}^{b l, \varepsilon}(x)=\Phi_{\varepsilon}(x)=\left(1-g\left(\frac{x^{\prime}}{\varepsilon}\right)-d\right) \frac{\partial u}{\partial x_{n}}(x)+\widehat{\phi}_{\varepsilon}(x) \quad \text { on } \Gamma_{\varepsilon},
$$

where, by a change of variables, $\widehat{\phi}_{\varepsilon}$ is defined by

$$
\widehat{\phi}_{\varepsilon}(x)=\varepsilon \int_{0}^{1-g\left(\frac{x^{\prime}}{\varepsilon}\right)}\left[g\left(\frac{x^{\prime}}{\varepsilon}\right)-1+\tau\right] \frac{\partial^{2} u}{\partial x_{n}^{2}}\left(x^{\prime}, 1-\varepsilon \tau\right) d \tau+\varepsilon \int_{0}^{1-g\left(\frac{x^{\prime}}{\varepsilon}\right)} \frac{\partial u_{0}^{b l}}{\partial x_{n}}\left(x^{\prime}, 1-\varepsilon \tau\right) d \tau .
$$

Since $u \in W^{3, \infty}(\Omega)$, it is easy to prove that

$$
\left\|\Phi_{\varepsilon}\right\|_{L^{\infty}\left(\Omega_{\varepsilon}\right)} \leq C \quad \text { and } \quad\left\|\nabla \Phi_{\varepsilon}\right\|_{L^{\infty}\left(\Omega_{\varepsilon}\right)^{n}} \leq \frac{C}{\varepsilon},
$$

hence, Lemma 4.2 implies $\left\|w_{0}^{b l, \varepsilon}\right\|_{H^{1}\left(\Omega_{\varepsilon}\right)} \leq C \varepsilon^{-1 / 2}$. On the other hand, we decompose $w_{0}^{b l, \varepsilon}(x)=w_{0,1}^{b l, \varepsilon}(x)+$ $w_{0,2}^{b l, \varepsilon}(x)$, where $w_{0,1}^{b l, \varepsilon}$ satisfies

$$
\begin{cases}-\operatorname{div} A_{\varepsilon} \nabla w_{0,1}^{b l, \varepsilon}=0 & \text { in } \Omega_{\varepsilon}, \\ w_{0,1}^{b l, \varepsilon}=\left[1-g\left(\frac{x^{\prime}}{\varepsilon}\right)-d\right] \frac{\partial u}{\partial x_{n}}\left(x^{\prime}, 1\right) & \text { on } \Gamma_{\varepsilon}, \\ w_{0,1}^{b l, \varepsilon}=0 & \text { on } \Gamma_{0}, \\ x^{\prime} \rightarrow w_{0,1}^{b l, \varepsilon}\left(x^{\prime}, x_{n}\right) & \text { periodic on } \Gamma_{\#},\end{cases}
$$

and $w_{0,2}^{b l, \varepsilon}$ satisfies

$$
\begin{cases}-\operatorname{div} A_{\varepsilon} \nabla w_{0,2}^{b l, \varepsilon}=0 & \text { in } \Omega_{\varepsilon}, \\ w_{0,2}^{b l, \varepsilon}=\widehat{\phi}_{\varepsilon} & \text { on } \Gamma_{\varepsilon}, \\ w_{0,2}^{b l, \varepsilon}=0 & \text { on } \Gamma_{0}, \\ x^{\prime} \rightarrow w_{0,2}^{b l, \varepsilon}\left(x^{\prime}, x_{n}\right) & \text { periodic on } \Gamma_{\#} .\end{cases}
$$

By Corollary 4.7, for any subset $\omega \subset \subset \Omega$, it follows that $\left\|w_{0,1}^{b l, \varepsilon}\right\|_{H^{1}(\omega)} \leq C \sqrt{\varepsilon}$. Since $\left\|\widehat{\phi}_{\varepsilon}\right\|_{L^{\infty}\left(\Omega_{\varepsilon}\right)} \leq C \varepsilon$ and $\left\|\nabla \widehat{\phi}_{\varepsilon}\right\|_{L^{\infty}\left(\Omega_{\varepsilon}\right)^{n}} \leq C$, Lemma 4.2 implies that $\left\|w_{0,2}^{b l, \varepsilon}\right\|_{H^{1}\left(\Omega_{\varepsilon}\right)} \leq C \sqrt{\varepsilon}$, which concludes the proof.

The final estimate for the zero-th order boundary layer is given in the following result.

Proposition 6.2. Let $u_{0}^{b l, \varepsilon}$ be defined by (6.1), then

$$
\left\|u_{0}^{b l, \varepsilon}\right\|_{H^{1}\left(\Omega_{\varepsilon}\right)} \leq C \sqrt{\varepsilon}
$$

and, for every open set $\omega \subset \subset \Omega$,

$$
\left\|u_{0}^{b l, \varepsilon}\right\|_{H^{1}(\omega)} \leq C \varepsilon
$$

Proof. This is a consequence of (6.5), (6.6) and of the previous lemma

$$
\left\|u_{0}^{b l, \varepsilon}\right\|_{H^{1}\left(\Omega_{\varepsilon}\right)} \leq \varepsilon\left(\left\|u_{0}^{b l}\right\|_{H^{1}\left(\Omega_{\varepsilon}\right)}+\left\|v_{0}^{b l, \varepsilon}\right\|_{H^{1}\left(\Omega_{\varepsilon}\right)}+\left\|w_{0}^{b l, \varepsilon}\right\|_{H^{1}\left(\Omega_{\varepsilon}\right)}\right) \leq C \varepsilon\left(1+1+\frac{1}{\sqrt{\varepsilon}}\right) \leq C \sqrt{\varepsilon}
$$


and

$$
\left\|u_{0}^{b l, \varepsilon}\right\|_{H^{1}(\omega)} \leq \varepsilon\left(\left\|u_{0}^{b l}\right\|_{H^{1}\left(\Omega_{\varepsilon}\right)}+\left\|v_{0}^{b l, \varepsilon}\right\|_{H^{1}\left(\Omega_{\varepsilon}\right)}+\left\|w_{0}^{b l, \varepsilon}\right\|_{H^{1}(\omega)}\right) \leq C \varepsilon(1+1+\sqrt{\varepsilon}) \leq C \varepsilon
$$

As a consequence of the study of the zero-th order boundary layer, we obtain the following error estimate for the homogenization of (4.1).

Theorem 6.3. Let $u_{\varepsilon}$ be the unique solution in $H\left(\Omega_{\varepsilon}\right)$ of (4.1) and $u$ be the unique solution in $H(\Omega)$ of (4.4). Assume that $u \in W^{3, \infty}(\Omega)$. Let $u_{1}(x, y)$ be defined by (2.5) (with any choice of the additive function $\widetilde{u}_{1}(x)$ ). Then

$$
\left\|u_{\varepsilon}(x)-u(x)-\varepsilon u_{1}\left(x, \frac{x}{\varepsilon}\right)\right\|_{H^{1}\left(\Omega_{\varepsilon}\right)} \leq C \sqrt{\varepsilon} .
$$

Proof. We observe that

$$
\left\|u_{\varepsilon}-u-\varepsilon u_{1}\right\|_{H^{1}\left(\Omega_{\varepsilon}\right)} \leq\left\|u_{\varepsilon}-u-u_{0}^{b l, \varepsilon}-\varepsilon u_{1}\right\|_{H^{1}\left(\Omega_{\varepsilon}\right)}+\left\|u_{0}^{b l, \varepsilon}\right\|_{H^{1}\left(\Omega_{\varepsilon}\right)} .
$$

Introducing the function

it clearly satisfies

$$
r_{\varepsilon}(x)=\frac{u_{\varepsilon}(x)-u(x)-u_{0}^{b l, \varepsilon}(x)-\varepsilon u_{1}\left(x, \frac{x}{\varepsilon}\right)}{\varepsilon}
$$

$$
\begin{cases}-\operatorname{div} A_{\varepsilon} \nabla r_{\varepsilon}=\frac{1}{\varepsilon}\left(f+\operatorname{div} A_{\varepsilon} \nabla u\right)+\operatorname{div} A_{\varepsilon} \nabla u_{1} & \text { in } \Omega_{\varepsilon}, \\ r_{\varepsilon}=-u_{1} & \text { on } \Gamma_{\varepsilon} \cup \Gamma_{0}, \\ x^{\prime} \rightarrow r_{\varepsilon}\left(x^{\prime}, x_{n}\right) & \text { periodic on } \Gamma_{\#} .\end{cases}
$$

We decompose $r_{\varepsilon}=r_{\varepsilon}^{1}+r_{\varepsilon}^{2}$, with $r_{\varepsilon}^{1}$ solution of

$$
\left\{\begin{aligned}
-\operatorname{div} A_{\varepsilon} \nabla r_{\varepsilon}^{1}=\frac{1}{\varepsilon}\left(f+\operatorname{div} A_{\varepsilon} \nabla u\right)+\operatorname{div} A_{\varepsilon} \nabla u_{1} & \text { in } \Omega_{\varepsilon}, \\
r_{\varepsilon}^{1}=0 & \text { on } \Gamma_{\varepsilon} \cup \Gamma_{0}, \\
x^{\prime} \rightarrow r_{\varepsilon}^{1}\left(x^{\prime}, x_{n}\right) & \text { periodic on } \Gamma_{\#} ;
\end{aligned}\right.
$$

and $r_{\varepsilon}^{2}$ solution of

$$
\left\{\begin{aligned}
-\operatorname{div} A_{\varepsilon} \nabla r_{\varepsilon}^{2}=0 & \text { in } \Omega_{\varepsilon}, \\
r_{\varepsilon}^{2}=-u_{1}=\chi^{j}\left(\frac{x}{\varepsilon}\right) \frac{\partial u}{\partial x_{j}}-\widetilde{u}_{1} & \text { on } \Gamma_{\varepsilon} \cup \Gamma_{0}, \\
x^{\prime} \rightarrow r_{\varepsilon}^{2}\left(x^{\prime}, x_{n}\right) & \text { periodic on } \Gamma_{\#} .
\end{aligned}\right.
$$

Since $\left\|u_{1}\right\|_{L^{\infty}\left(\Omega_{\varepsilon}\right)} \leq C$ and $\left\|\nabla u_{1}\right\|_{L^{\infty}\left(\Omega_{\varepsilon}\right)^{n}} \leq C \varepsilon^{-1}$, by Lemma 4.2 , it follows that $\left\|r_{\varepsilon}^{2}\right\|_{H^{1}\left(\Omega_{\varepsilon}\right)} \leq C \varepsilon^{-1 / 2}$. On the other hand, $r_{\varepsilon}^{1}$ satisfies

$$
\left\|r_{\varepsilon}^{1}\right\|_{H\left(\Omega_{\varepsilon}\right)}^{2} \leq \frac{1}{\lambda}\left|\int_{\Omega_{\varepsilon}}\left[\frac{1}{\varepsilon}\left(f+\operatorname{div} A_{\varepsilon} \nabla u\right)+\operatorname{div} A_{\varepsilon} \nabla u_{1}\right] r_{\varepsilon}^{1} d x\right| .
$$

Recalling the proof of Theorems 2.2 and 3.7, it is easy to see that, for any $\phi \in H\left(\Omega_{\varepsilon}\right)$, we obtain

$$
\left|\int_{\Omega_{\varepsilon}}\left[\frac{1}{\varepsilon}\left(f+\operatorname{div} A_{\varepsilon} \nabla u\right)+\operatorname{div} A_{\varepsilon} \nabla u_{1}\right] \phi d x\right| \leq C\|\phi\|_{H\left(\Omega_{\varepsilon}\right)} .
$$

Therefore $\left\|r_{\varepsilon}^{1}\right\|_{H\left(\Omega_{\varepsilon}\right)} \leq C$. As a consequence

$$
\left\|u_{\varepsilon}-u-u_{0}^{b l, \varepsilon}-\varepsilon u_{1}\right\|_{H^{1}\left(\Omega_{\varepsilon}\right)} \leq \varepsilon\left[\left\|r_{\varepsilon}^{1}\right\|_{H\left(\Omega_{\varepsilon}\right)}+\left\|r_{\varepsilon}^{2}\right\|_{H^{1}\left(\Omega_{\varepsilon}\right)}\right] \leq C \sqrt{\varepsilon} .
$$


Hence $(6.11,6.12)$ and $(6.9)$ yield the desired result.

We now study the first-order boundary layer $u_{1}^{b l, \varepsilon}$ defined by (4.3) (this will allow us to improve Th. 6.3). We denote by $d_{j}^{*}$ (respectively $d_{j}^{0}$ ) the boundary layer tail associated to the side $\Gamma$ (respectively $\Gamma_{0}$ ) and to $h\left(\frac{x}{\varepsilon}\right)=\chi^{j}\left(\frac{x}{\varepsilon}\right)$, as defined in Lemma 4.4. We decompose $u_{1}^{b l, \varepsilon}(x)=v_{1}^{b l, \varepsilon}(x)+w_{1}^{b l, \varepsilon}(x)$, where $v_{1}^{b l, \varepsilon}$ satisfies

$$
\left\{\begin{array}{cl}
-\operatorname{div} A_{\varepsilon} \nabla v_{1}^{b l, \varepsilon}=0 & \text { in } \Omega_{\varepsilon} ; \\
v_{1}^{b l, \varepsilon}(x)=d_{j}^{0} \frac{\partial u}{\partial x_{j}}(x)-\widetilde{u}_{1}(x) & \text { on } \Gamma_{0} ; \\
v_{1}^{b l, \varepsilon}(x)=d_{j}^{*} \frac{\partial u}{\partial x_{j}}(x)-\widetilde{u}_{1}(x) & \text { on } \Gamma_{\varepsilon} ; \\
x^{\prime} \rightarrow v_{1}^{b l, \varepsilon}\left(x^{\prime}, x_{n}\right) & \text { periodic on } \Gamma_{\#} ;
\end{array}\right.
$$

and $w_{1}^{b l, \varepsilon}$ satisfies

$$
\left\{\begin{array}{cl}
-\operatorname{div} A_{\varepsilon} \nabla w_{1}^{b l, \varepsilon}=0 & \text { in } \Omega_{\varepsilon} ; \\
w_{1}^{b l, \varepsilon}(x)=\left(\chi^{j}\left(\frac{x}{\varepsilon}\right)-d_{j}^{0}\right) \frac{\partial u}{\partial x_{j}}(x) & \text { on } \Gamma_{0} ; \\
w_{1}^{b l, \varepsilon}(x)=\left(\chi^{j}\left(\frac{x}{\varepsilon}\right)-d_{j}^{*}\right) \frac{\partial u}{\partial x_{j}}(x) & \text { on } \Gamma_{\varepsilon} ; \\
x^{\prime} \rightarrow w_{1}^{b l, \varepsilon}\left(x^{\prime}, x_{n}\right) & \text { periodic on } \Gamma_{\#} .
\end{array}\right.
$$

By using Lemma 4.6 and Corollary 4.7, on the behavior of boundary layers, we are going to show that $w_{1}^{b l, \varepsilon}$ converges strongly to 0 in the interior of $\Omega_{\varepsilon}$. Remark that we shall use these results twice : first for studying the boundary layer associated to $\Gamma_{\varepsilon}$, second for that associated to $\Gamma_{0}$ (where $\Gamma_{0}$, being fixed, is a very special case of an oscillating boundary). On the other hand the homogenization of $v_{1}^{b l, \varepsilon}$ is similar to that of $u_{\varepsilon}$, solution of (4.1), as described in Theorem 6.3. Formally, it admits the following asymptotic expansion

$$
v_{1}^{b l, \varepsilon}(x)=v_{1}^{b l}(x)+\varepsilon v_{1,1}^{b l}\left(x, \frac{x}{\varepsilon}\right)+\ldots
$$

where $v_{1}^{b l}(x)$ satisfies

$$
\begin{cases}-\operatorname{div} A^{*} \nabla v_{1}^{b l}=0 & \text { in } \Omega ; \\ v_{1}^{b l}(x)=d_{j}^{0} \frac{\partial u}{\partial x_{j}}(x)-\widetilde{u}_{1}(x) & \text { on } \Gamma_{0} \\ v_{1}^{b l}(x)=d_{j}^{*} \frac{\partial u}{\partial x_{j}}(x)-\widetilde{u}_{1}(x) & \text { on } \Gamma ; \\ x^{\prime} \rightarrow v_{1}^{b l}\left(x^{\prime}, x_{n}\right) & \text { periodic on } \Gamma_{\#}\end{cases}
$$

and $v_{1,1}^{b l}\left(x, \frac{x}{\varepsilon}\right)=\chi^{j}\left(\frac{x}{\varepsilon}\right) \frac{\partial v_{1}^{b l}}{\partial x_{j}}(x)$. The asymptotic behavior of $u_{1}^{b l, \varepsilon}, v_{1}^{b l, \varepsilon}, w_{1}^{b l, \varepsilon}$ is given by the next result.

Lemma 6.4. For every open set $\omega \subset \subset \Omega, v_{1}^{b l, \varepsilon} \rightarrow v_{1}^{b l}$ weakly in $H^{1}(\omega), w_{1}^{b l, \varepsilon} \rightarrow 0$ strongly in $H^{1}(\omega)$, and consequently $u_{1}^{b l, \varepsilon} \rightarrow v_{1}^{b l}$ weakly in $H^{1}(\omega)$. Furthermore, there exists a constant $C>0$ such that

$$
\left\|u_{1}^{b l, \varepsilon}(x)-v_{1}^{b l}(x)-\varepsilon v_{1,1}^{b l}\left(x, \frac{x}{\varepsilon}\right)\right\|_{H^{1}(\omega)} \leq C \sqrt{\varepsilon}
$$

and

$$
\left\|u_{1}^{b l, \varepsilon}(x)-v_{1}^{b l}(x)\right\|_{L^{2}(\omega)} \leq C \sqrt{\varepsilon}
$$


Proof. Let $\omega \subset \subset \Omega$. The proof that $v_{1}^{b l, \varepsilon} \rightarrow v_{1}^{b l}$ weakly in $H^{1}(\omega)$ is completely standard, and the strong convergence of $w_{1}^{b l, \varepsilon}$ to 0 in $H^{1}(\omega)$ is a direct consequence of Corollary 4.7. It remains to establish the error estimates. For sufficiently small $\varepsilon$, we have $\omega \subset \subset \Omega_{\varepsilon} \subset \Omega$. Thus

$$
\left\|u_{1}^{b l, \varepsilon}-v_{1}^{b l}-\varepsilon v_{1,1}^{b l}\right\|_{H^{1}(\omega)} \leq\left\|v_{1}^{b l, \varepsilon}-v_{1}^{b l}-\varepsilon v_{1,1}^{b l}\right\|_{H^{1}\left(\Omega_{\varepsilon}\right)}+\left\|w_{1}^{b l, \varepsilon}\right\|_{H^{1}(\omega)} .
$$

We decompose $w_{1}^{b l, \varepsilon}=w_{1,0}^{b l, \varepsilon}+w_{1, *}^{b l, \varepsilon}$ with

$$
\begin{cases}-\operatorname{div} A_{\varepsilon} \nabla w_{1,0}^{b l, \varepsilon}=0 & \text { in } \Omega_{\varepsilon} ; \\ w_{1,0}^{b l, \varepsilon}(x)=\left(\chi^{j}\left(\frac{x}{\varepsilon}\right)-d_{j}^{0}\right) \frac{\partial u}{\partial x_{j}}(x) & \text { on } \Gamma_{0} ; \\ w_{1,0}^{b l, \varepsilon}=0 & \text { on } \Gamma_{\varepsilon} ; \\ x^{\prime} \rightarrow w_{1,0}^{b l, \varepsilon}\left(x^{\prime}, x_{n}\right) & \text { periodic on } \Gamma_{\#} ;\end{cases}
$$

and

$$
\left\{\begin{array}{cl}
-\operatorname{div} A_{\varepsilon} \nabla w_{1, *}^{b l, \varepsilon}=0 & \text { in } \Omega_{\varepsilon} ; \\
w_{1, *}^{b l, \varepsilon}(x)=\left(\chi^{j}\left(\frac{x}{\varepsilon}\right)-d_{j}^{*}\right) \frac{\partial u}{\partial x_{j}}(x) & \text { on } \Gamma_{\varepsilon} ; \\
w_{1, *}^{b l, \varepsilon}=0 & \text { on } \Gamma_{0}, \\
x^{\prime} \rightarrow w_{1, *}^{b l, \varepsilon}\left(x^{\prime}, x_{n}\right) & \text { periodic on } \Gamma_{\#} \cdot
\end{array}\right.
$$

Applying Lemma 4.6 and Corollary 4.7 to both terms $w_{1,0}^{b l, \varepsilon}$ and $w_{1, *}^{b l, \varepsilon}$, we obtain that

$$
\left\|w_{1}^{b l, \varepsilon}\right\|_{H^{1}(\omega)} \leq C \sqrt{\varepsilon}
$$

On the other hand, we apply Theorem 6.3 , with $u_{\varepsilon}, u$ and $u_{1}$ replaced by $v_{1}^{b l, \varepsilon}, v_{1}^{b l}$ and $v_{1,1}^{b l}$ respectively, and take $f=0$ along with the non-homogeneous boundary data given in (6.14). It yields

$$
\left\|v_{1}^{b l, \varepsilon}-v_{1}^{b l}-\varepsilon v_{1,1}^{b l}\right\|_{H^{1}\left(\Omega_{\varepsilon}\right)} \leq C \sqrt{\varepsilon} .
$$

Combining $(6.15,6.16)$ and $(6.17)$ leads to $(i)$. Finally, $(i i)$ is deduced from $(i)$ by noticing that

$$
\left\|u_{1}^{b l, \varepsilon}-v_{1}^{b l}\right\|_{L^{2}(\omega)} \leq\left\|u_{1}^{b l, \varepsilon}-v_{1}^{b l}-\varepsilon v_{1,1}^{b l}\right\|_{L^{2}(\omega)}+\varepsilon\left\|v_{1,1}^{b l}\right\|_{L^{2}\left(\Omega_{\varepsilon}\right)} \leq C(\sqrt{\varepsilon}+\varepsilon) .
$$

We now improve Theorem 6.3 , by adding the zero and first order boundary layers.

Theorem 6.5. Let $u_{\varepsilon}$ be the unique solution in $H\left(\Omega_{\varepsilon}\right)$ of (4.1) and $u$ be the unique solution in $H(\Omega)$ of (4.4). Assume that $u \in W^{3, \infty}(\Omega)$. Let $u_{1}$ be defined by (2.5). Then

$$
\begin{aligned}
& \text { (i) }\left\|u_{\varepsilon}(x)-u(x)-u_{0}^{b l, \varepsilon}(x)-\varepsilon u_{1}\left(x, \frac{x}{\varepsilon}\right)-\varepsilon u_{1}^{b l, \varepsilon}(x)\right\|_{H^{1}\left(\Omega_{\varepsilon}\right)} \leq C \varepsilon ; \\
& (i i)\left\|u_{\varepsilon}(x)-u(x)-\varepsilon u_{1}\left(x, \frac{x}{\varepsilon}\right)\right\|_{H^{1}(\omega)} \leq C \varepsilon ;
\end{aligned}
$$

where in (ii) the constant $C$ depends only on $\omega$ which is any open set such that $\omega \subset \subset \Omega$. 
Proof. Let us define the function

$$
r_{\varepsilon}(x)=\frac{u_{\varepsilon}(x)-u(x)-u_{0}^{b l, \varepsilon}(x)-\varepsilon u_{1}\left(x, \frac{x}{\varepsilon}\right)-\varepsilon u_{1}^{b l, \varepsilon}(x)}{\varepsilon} .
$$

We proceed exactly in the same way as in the proof of Theorem 6.3 . Observing that, in this case, $r_{\varepsilon}=r_{\varepsilon}^{1}$ and $r_{\varepsilon}^{2}=0$ immediately leads to $(i)$. By $(i),(6.10)$ and $(i)$ of Lemma 6.4 , we have

$$
\begin{aligned}
\left\|u_{\varepsilon}-u-\varepsilon u_{1}\right\|_{H^{1}(\omega)} & \leq\left\|u_{\varepsilon}-u-u_{0}^{b l, \varepsilon}-\varepsilon u_{1}-\varepsilon u_{1}^{b l, \varepsilon}\right\|_{H^{1}\left(\Omega_{\varepsilon}\right)}+\left\|u_{0}^{b l, \varepsilon}\right\|_{H^{1}(\omega)}+\varepsilon\left\|u_{1}^{b l, \varepsilon}\right\|_{H^{1}(\omega)} \\
& \leq C \varepsilon+C \varepsilon+\varepsilon\left\|u_{1}^{b l, \varepsilon}-v_{1}^{b l}-\varepsilon v_{1,1}^{b l}\right\|_{H^{1}(\omega)}+\varepsilon\left\|v_{1}^{b l}+\varepsilon v_{1,1}^{b l}\right\|_{H^{1}\left(\Omega_{\varepsilon}\right)} \leq C \varepsilon
\end{aligned}
$$

which is nothing but $(i i)$.

Theorem 6.5 can be improved by adding the next corrector term $u_{2}$ and by removing all the non-explicit boundary layer terms, at least in the interior of the domain.

Theorem 6.6. Let $u_{\varepsilon}$ be the unique solution in $H\left(\Omega_{\varepsilon}\right)$ of (4.1) and $u$ be the unique solution in $H(\Omega)$ of (4.4). Assume that $u \in W^{3, \infty}(\Omega)$. Let $u_{1}, u_{2}, u_{0}^{b l, \varepsilon}, u_{1}^{b l, \varepsilon}$ be given by (4.2). Then,

$$
\left\|u_{\varepsilon}(x)-u(x)-u_{0}^{b l, \varepsilon}(x)-\varepsilon u_{1}\left(x, \frac{x}{\varepsilon}\right)-\varepsilon u_{1}^{b l, \varepsilon}(x)-\varepsilon^{2} u_{2}\left(x, \frac{x}{\varepsilon}\right)\right\|_{H^{1}\left(\Omega_{\varepsilon}\right)} \leq C \varepsilon^{\frac{3}{2}}
$$

and

$$
\left\|u_{\varepsilon}(x)-u(x)-\varepsilon u_{0}^{b l}(x)-\varepsilon u_{1}\left(x, \frac{x}{\varepsilon}\right)-\varepsilon\left(v_{1}^{b l}(x)+\varepsilon v_{1,1}^{b l}\left(x, \frac{x}{\varepsilon}\right)\right)-\varepsilon^{2} u_{2}\left(x, \frac{x}{\varepsilon}\right)\right\|_{H^{1}(\omega)} \leq C \varepsilon^{\frac{3}{2}} .
$$

To prove Theorem 6.6 we need a preliminary lemma concerning the asymptotic behavior of $v_{0}^{b l, \varepsilon}$, defined by (6.3), which is part of the zero order boundary layer. Theorems 6.3 and 6.5 will be the main tools in order to prove that, actually, $v_{0}^{b l, \varepsilon}$ tends to zero and to evaluate the rate of convergence. The following result clearly improve estimate (6.6).

Lemma 6.7. Let $v_{0}^{b l, \varepsilon}$ be defined in (6.3). Then

$$
\left\|v_{0}^{b l, \varepsilon}\right\|_{H^{1}\left(\Omega_{\varepsilon}\right)} \leq C \sqrt{\varepsilon}
$$

and, for every open set $\omega \subset \subset \Omega$, there exists a positive constant $C>0$, depending on $\omega$ but not on $\varepsilon$, such that

$$
\left\|v_{0}^{b l, \varepsilon}\right\|_{H^{1}(\omega)} \leq C \varepsilon
$$

Proof. Denoting by $v_{0}^{b l}(x)$ and by $v_{0,1}^{b l}(x, y)$ the limit and the first order corrector of $v_{0}^{b l, \varepsilon}(x)$, respectively, it is easy to see that the homogenized problem for $v_{0}^{b l}$ is (recall (6.3) and (6.2))

$$
\begin{cases}-\operatorname{div} A^{*} \nabla v_{0}^{b l}=\operatorname{div} A^{*} \nabla u_{0}^{b l}=0 & \text { in } \Omega, \\ v_{0}^{b l}=0 & \text { on } \Gamma \cup \Gamma_{0}, \\ x^{\prime} \rightarrow v_{0}^{b l}\left(x^{\prime}, x_{n}\right) & \text { periodic on } \Gamma_{\#} .\end{cases}
$$

Thus $v_{0}^{b l} \equiv 0$ and therefore $v_{0,1}^{b l}(x, y)=\chi^{j}(y) \frac{\partial v_{0}^{b l}}{\partial x_{j}}(x) \equiv 0$. Hence, the thesis follows by Theorem 6.3 and $(i i)$ of Theorem 6.5 , with $u_{\varepsilon}, u$ and $u_{1}$ replaced by $v_{0}^{b l, \varepsilon}, v_{0}^{b l}$ and $v_{0,1}^{b l}$ respectively. 
We now address the proof of Theorem 6.6.

Proof. Set

$$
r_{\varepsilon}(x)=\frac{u_{\varepsilon}(x)-u(x)-u_{0}^{b l, \varepsilon}(x)-\varepsilon u_{1}\left(x, \frac{x}{\varepsilon}\right)-\varepsilon u_{1}^{b l, \varepsilon}(x)-\varepsilon^{2} u_{2}\left(x, \frac{x}{\varepsilon}\right)}{\varepsilon^{2}} ;
$$

clearly, $r_{\varepsilon}$ satisfies

$$
\begin{cases}-\operatorname{div} A_{\varepsilon} \nabla r_{\varepsilon}=\frac{1}{\varepsilon^{2}}\left(f+\operatorname{div} A_{\varepsilon} \nabla u\right)+\frac{1}{\varepsilon} \operatorname{div} A_{\varepsilon} \nabla u_{1}+\operatorname{div} A_{\varepsilon} \nabla u_{2} & \text { in } \Omega_{\varepsilon}, \\ r_{\varepsilon}=-u_{2} & \text { on } \Gamma_{\varepsilon} \cup \Gamma_{0}, \\ x^{\prime} \rightarrow r_{\varepsilon}\left(x^{\prime}, x_{n}\right) & \text { periodic on } \Gamma_{\#} .\end{cases}
$$

We decompose $r_{\varepsilon}=r_{\varepsilon}^{1}+r_{\varepsilon}^{2}$, where $r_{\varepsilon}^{1}$ satisfies

$$
\begin{cases}-\operatorname{div} A_{\varepsilon} \nabla r_{\varepsilon}^{1}=\frac{1}{\varepsilon^{2}}\left(f+\operatorname{div} A_{\varepsilon} \nabla u\right)+\frac{1}{\varepsilon} \operatorname{div} A_{\varepsilon} \nabla u_{1}+\operatorname{div} A_{\varepsilon} \nabla u_{2} & \text { in } \Omega_{\varepsilon}, \\ r_{\varepsilon}^{1}=0 & \text { on } \Gamma_{\varepsilon} \cup \Gamma_{0}, \\ x^{\prime} \rightarrow r_{\varepsilon}^{1}\left(x^{\prime}, x_{n}\right) & \text { periodic on } \Gamma_{\#},\end{cases}
$$

and $r_{\varepsilon}^{2}$ satisfies

$$
\begin{cases}-\operatorname{div} A_{\varepsilon} \nabla r_{\varepsilon}^{2}=0 & \text { in } \Omega_{\varepsilon}, \\ r_{\varepsilon}^{2}=-u_{2}=-\chi^{i j} \frac{\partial^{2} u}{\partial x_{i} \partial x_{j}}+\chi^{j} \frac{\partial \widetilde{u}_{1}}{\partial x_{j}}-\widetilde{u}_{2} & \text { on } \Gamma_{\varepsilon} \cup \Gamma_{0}, \\ x^{\prime} \rightarrow r_{\varepsilon}^{2}\left(x^{\prime}, x_{n}\right) & \text { periodic on } \Gamma_{\#} .\end{cases}
$$

By Lemma 4.2, it follows that $\left\|r_{\varepsilon}^{2}\right\|_{H^{1}\left(\Omega_{\varepsilon}\right)} \leq C \varepsilon^{-1 / 2}$. On the other hand, we have

$$
\left\|r_{\varepsilon}^{1}\right\|_{H\left(\Omega_{\varepsilon}\right)} \leq \frac{1}{\lambda}\left\|\frac{1}{\varepsilon^{2}}\left(f+\operatorname{div} A_{\varepsilon} \nabla u\right)+\frac{1}{\varepsilon} \operatorname{div} A_{\varepsilon} \nabla u_{1}+\operatorname{div} A_{\varepsilon} \nabla u_{2}\right\|_{H^{\prime}\left(\Omega_{\varepsilon}\right)} \leq C,
$$

where the last inequality is obtained as in the proof of Theorem 6.3, using the equations for $u, u_{1}, u_{2}$ and $u_{3}$ as it is stated at the beginning of Section 4 (including the Eq. (2.10) for $\widetilde{u}_{1}$ ). Therefore, we obtain (i), i.e.

$$
\left\|u_{\varepsilon}-u-u_{0}^{b l, \varepsilon}-\varepsilon u^{1}-\varepsilon u_{1}^{b l, \varepsilon}-\varepsilon^{2} u_{2}\right\|_{H^{1}\left(\Omega_{\varepsilon}\right)}=\varepsilon^{2}\left\|r_{\varepsilon}\right\|_{H^{1}\left(\Omega_{\varepsilon}\right)} \leq C \varepsilon^{\frac{3}{2}} .
$$

Let us prove now $(i i)$. Let $\omega \subset \subset \Omega$. For sufficiently small $\varepsilon$, we have $\omega \subset \subset \Omega_{\varepsilon} \subset \Omega$. We observe that

$$
\begin{aligned}
& \left\|u_{\varepsilon}-u-\varepsilon u_{0}^{b l}-\varepsilon u_{1}-\varepsilon\left(v_{1}^{b l}+\varepsilon v_{1,1}^{b l}\right)-\varepsilon^{2} u_{2}\right\|_{H^{1}(\omega)} \leq\left\|u_{\varepsilon}-u-u_{0}^{b l, \varepsilon}-\varepsilon u_{1}-\varepsilon u_{1}^{b l, \varepsilon}-\varepsilon^{2} u_{2}\right\|_{H^{1}\left(\Omega_{\varepsilon}\right)} \\
& +\varepsilon\left\|v_{0}^{b l, \varepsilon}\right\|_{H^{1}(\omega)}+\varepsilon\left\|w_{0}^{b l, \varepsilon}\right\|_{H^{1}(\omega)}+\varepsilon\left\|u_{1}^{b l, \varepsilon}-v_{1}^{b l}-\varepsilon v_{1,1}^{b l}\right\|_{H^{1}(\omega)} .
\end{aligned}
$$

By Lemma 6.7, (6.8) and (i) of Lemma 6.4, the last three terms in the previous inequality are bounded by $C \varepsilon^{\frac{3}{2}}$, which immediately yields $(i i)$.

We are now in a position to prove the main result of this section.

Theorem 6.8. Let $u_{\varepsilon} \in H\left(\Omega_{\varepsilon}\right)$ and $u \in H(\Omega)$ be the unique solutions of (4.1) and (4.4) respectively. Assume that $u \in W^{3, \infty}(\Omega)$. Let $u_{1}$ be defined by

$$
u_{1}\left(x, \frac{x}{\varepsilon}\right)=-\chi^{j}\left(\frac{x}{\varepsilon}\right) \frac{\partial u}{\partial x_{j}}(x)+\widetilde{u}_{1}(x),
$$


where $\widetilde{u}_{1}$ is the unique solution of

$$
\begin{cases}-\operatorname{div} A^{*} \nabla \widetilde{u}_{1}=c_{i j k} \frac{\partial^{3} u}{\partial x_{i} \partial x_{j} \partial x_{k}} & \text { in } \Omega ; \\ \widetilde{u}_{1}=d_{j}^{0} \frac{\partial u}{\partial x_{j}} & \text { on } \Gamma_{0} ; \\ \widetilde{u}_{1}=d_{j}^{*} \frac{\partial u}{\partial x_{j}} & \text { on } \Gamma ; \\ x^{\prime} \rightarrow \widetilde{u}_{1}\left(x^{\prime}, x_{n}\right) & \text { periodic on } \Gamma_{\#} ;\end{cases}
$$

with the constants $d_{j}^{*}$ (respectively $d_{j}^{0}$ ) defined as the boundary layer tails associated to the side $\Gamma$ (respectively $\left.\Gamma_{0}\right)$ and to $h(y)=\chi^{j}(y)$ (cf. Lemma 4.4), and

$$
c_{i j k}=\int_{Y}\left[a_{k l}(y) \frac{\partial \chi^{i j}}{\partial y_{l}}(y)-a_{i j}(y) \chi^{k}(y)\right] d y .
$$

Finally, for sufficiently small $\varepsilon$, let $\bar{u}_{\varepsilon}$ be the unique solution in $H(\Omega)$ of

$$
\begin{cases}-\operatorname{div} A^{*} \nabla \bar{u}_{\varepsilon}=f & \text { in } \Omega ; \\ \bar{u}_{\varepsilon}=\varepsilon d \frac{\partial \bar{u}_{\varepsilon}}{\partial x_{n}} & \text { on } \Gamma ; \\ \bar{u}_{\varepsilon}=0 & \text { on } \Gamma_{0} ; \\ x^{\prime} \rightarrow \bar{u}_{\varepsilon}\left(x^{\prime}, x_{n}\right) & \text { periodic on } \Gamma_{\#},\end{cases}
$$

where the constant $d$ is the boundary layer tail defined by Lemma 4.4 applied to $h(y)=1-g(y)$. Then, for any open set $\omega \subset \subset \Omega$, there exists a positive constant $C>0$, depending on $\omega$ but not on $\varepsilon$, such that

$$
\left\|u_{\varepsilon}(x)-\bar{u}_{\varepsilon}(x)-\varepsilon u_{1}\left(x, \frac{x}{\varepsilon}\right)-\varepsilon^{2} u_{2}\left(x, \frac{x}{\varepsilon}\right)\right\|_{H^{1}(\omega)} \leq C \varepsilon^{\frac{3}{2}},
$$

and

$$
\left\|u_{\varepsilon}(x)-\bar{u}_{\varepsilon}(x)-\varepsilon u_{1}\left(x, \frac{x}{\varepsilon}\right)\right\|_{L^{2}(\omega)} \leq C \varepsilon^{\frac{3}{2}}
$$

Proof. Since $\tilde{u}_{1}$ satisfied the boundary condition in (6.18), it follows from (6.14) that $v_{1}^{b l}=0$ and therefore $v_{1,1}^{b l}=0$. Hence, by $(i i)$ of Theorem 6.6 , it follows that

$$
\left\|u_{\varepsilon}-u-\varepsilon u_{0}^{b l}-\varepsilon u_{1}-\varepsilon^{2} u_{2}\right\|_{H^{1}(\omega)} \leq C \varepsilon^{\frac{3}{2}}
$$

Now let us consider the function $\vartheta_{\varepsilon}=u+\varepsilon u_{0}^{b l}$. As in Remarks 5.4 and 3.11, it is not difficult to see that $\bar{u}_{\varepsilon}$ differs from $\vartheta_{\varepsilon}$ by a term of order $\varepsilon^{2}$ in the $H^{1}(\Omega)$-norm, then we may substitute $u+\varepsilon u_{0}^{b l}=\vartheta_{\varepsilon}$ with $\bar{u}_{\varepsilon}$ in the previous inequality to obtain (6.20). Finally (6.21) is a direct consequence of (6.20).

Remark 6.9. The interior error estimates of Theorem 6.8 require the knowledge of the boundary layer tails $d_{j}^{0}, d_{j}^{*}$ and $d$ which are crucial in the definition of $\widetilde{u}_{1}$ and $\bar{u}_{\varepsilon}$. Remark that, if we choose other boundary conditions for $\widetilde{u}_{1}$ and $\bar{u}_{\varepsilon}$, then boundary layers would automatically appear explicitly in the error estimates. On the contrary, with a right choice of boundary conditions, Theorem 6.8 states that only the boundary layer tails are necessary for deriving interior error estimates. Remark that Theorem 6.8 holds true also for systems of equations. Estimate (6.21) improves greatly the following deceptive estimate

$$
\left\|u_{\varepsilon}(x)-u(x)\right\|_{L^{2}(\omega)} \leq C \sqrt{\varepsilon}
$$


furnished by Theorem 6.3. To investigate the effects on the homogenized limit of adding corrector terms in (6.21), it is instructive to introduce a new function $\widetilde{u}_{\varepsilon}$ defined by

$$
\widetilde{u}_{\varepsilon}(x)=\bar{u}_{\varepsilon}(x)+\varepsilon \widetilde{u}_{1}(x) .
$$

Then, formally $\widetilde{u}_{\varepsilon}$ is a solution of

$$
\begin{cases}-\operatorname{div} A^{*} \nabla \widetilde{u}_{\varepsilon}-\varepsilon c_{i j k} \frac{\partial^{3} \widetilde{u}_{\varepsilon}}{\partial x_{i} \partial x_{j} \partial x_{k}}=f+\mathcal{O}\left(\varepsilon^{2}\right) & \text { in } \Omega \\ \widetilde{u}_{\varepsilon}=\varepsilon d_{j}^{0} \frac{\partial \widetilde{u}_{\varepsilon}}{\partial x_{j}}+\varepsilon d \frac{\partial \widetilde{u}_{\varepsilon}}{\partial x_{n}}+\mathcal{O}\left(\varepsilon^{2}\right) & \text { on } \Gamma ; \\ \widetilde{u}_{\varepsilon}=\varepsilon d_{j}^{*} \frac{\partial \widetilde{u}_{\varepsilon}}{\partial x_{j}}+\mathcal{O}\left(\varepsilon^{2}\right) & \text { on } \Gamma_{0} ; \\ x^{\prime} \rightarrow \widetilde{u}_{\varepsilon}\left(x^{\prime}, x_{n}\right) & \text { periodic on } \Gamma_{\#} ;\end{cases}
$$

where $\mathcal{O}\left(\varepsilon^{2}\right)$ denotes remaider terms of order $\varepsilon^{2}$ in both $L^{2}(\Omega)$ and $H^{1}(\Omega)$ norms. Remark that the dependence on $\varepsilon$ in $\widetilde{u}_{\varepsilon}$ is explicit (on the contrary of $u_{\varepsilon}$ ) and that it is not an oscillating function. Clearly, equation (6.22) displays dispersive effects and an effective Fourier boundary condition as a consequence of the boundary layer tails.

Remark 6.10. To conclude the paper, we conjecture that all the results in Sections 4,5 and 6 hold also if we substitute the periodic conditions on the lateral boundaries with Dirichlet boundary conditions everywhere. It may however be necessary to introduce boundary layers at the corners of the domain. This fact could be a very technical point; in order to avoid such technicalities, we have prefered to treat a periodic domain in this paper.

The second author would like to thank the Laboratoire d'Analyse Numérique, at Paris 6 University, where this work began during a visit supported by a C.N.R. fellowship.

\section{REFERENCES}

[1] T. Abboud and H. Ammari, Diffraction at a curved grating, TM and TE cases, homogenization. J. Math. Anal. Appl. 202 (1996) 995-1206.

[2] Y. Achdou, Effect d'un revêtement métallisé mince sur la réflexion d'une onde électromagnétique. C.R. Acad. Sci. Paris Sér. I Math. 314 (1992) 217-222.

[3] Y. Achdou and O. Pironneau, Domain decomposition and wall laws. C.R. Acad. Sci. Paris Sér. I Math. 320 (1995) $541-547$.

[4] Y. Achdou and O. Pironneau, A 2nd order condition for flow over rough walls, in Proc. Int. Conf. on Nonlinear Diff. Eqs. and Appl., Bangalore, Shrikant Ed. (1996).

[5] G. Allaire and G. Bal, Homogenization of the criticality spectral equation in neutron transport. M2AN to appear.

[6] G. Allaire and C. Conca, Bloch wave homogenization and spectral asymptotic analysis. J. Math. Pures et Appl. 77 (1998) 153-208.

[7] G. Allaire and C. Conca, Boundary layers in the homogenization of a spectral problem in fluid-solid structures. SIAM J. Math. Anal. 29 (1998) 343-379.

[8] M. Avellaneda and F.-H. Lin, Homogenization of elliptic problems with L ${ }^{\mathrm{p}}$ boundary data. Appl. Math. Optim. 15 (1987) 93-107.

[9] M. Avellaneda and F.-H. Lin, Compactness methods in the theory of homogenization. C.P.A.M., XL (1987) 803-847.

[10] I. Babuška, Solution of interface problems by homogenization I, II, III. SIAM J. Math. Anal. 7 (1976) 603-634 and 635-645; 8 (1977) 923-937.

[11] N. Bakhvalov and G. Panasenko, Homogenization, averaging processes in periodic media. Kluwer Academic Publishers, Dordrecht, Mathematics and its Applications 36 (1990).

[12] G. Bal, First-order corrector for the homogenization of the criticality eigenvalue problem in the even parity formulation of the neutron transport, to appear.

[13] A. Bensoussan, J.L. Lions and G. Papanicolaou, Asymptotic analysis for periodic structures. North Holland, Amsterdam (1978).

[14] A. Bensoussan, J.L. Lions and G. Papanicolaou, Boundary layers and homogenization of transport processes. Publ. Res. Inst. Math. Sci. 15 (1979) 53-157. 
[15] A. Bourgeat and E. Marusic-Paloka, Non-linear effects for flow in periodically constricted channel caused by high injection rate. Mathematical Models and Methods in Applied Sciences 8 (1998) 379-405.

[16] R. Brizzi and J.P. Chalot, Homogénéisation de frontière. PhD Thesis, Université de Nice (1978).

[17] G. Buttazzo and R.V. Kohn, Reinforcement by a thin layer with oscillating thickness. Appl. Math. Optim. 16 (1987) $247-261$.

[18] G. Chechkin, A. Friedman and A. Piatnitski, The boundary-value problem in domains with very rapidly oscillating boundary. INRIA Report 3062 (1996).

[19] R. Dautray and J.-L. Lions, Analyse mathématique et calcul numérique, Tome 3, Masson, Paris (1984).

[20] B. Engquist and J.C. Nédélec, Effective boundary conditions for accoustic and electro-magnetic scaterring in thin layers. Internal report 278, CMAP École Polytechnique (1993).

[21] A. Friedman, B. Hu and Y. Liu, A boundary value problem for the Poisson equation with multi-scale oscillating boundary. $J$. Diff. Eq. 137 (1997) 54-93.

[22] W. Jäger and A. Mikelic, On the boundary conditions at the contact interface between a porous medium and a free fluid. Ann. Scuola Norm. Sup. Pisa Cl. Sci. 23 (1996) 403-465.

[23] E. Landis and G. Panasenko, A theorem on the asymptotics of solutions of elliptic equations with coefficients periodic in all variables except one. Soviet Math. Dokl. 18 (1977) 1140-1143.

[24] J.L. Lions, Some methods in the mathematical analysis of systems and their controls. Science Press, Beijing, Gordon and Breach, New York (1981).

[25] S. Moskow and M. Vogelius, First order corrections to the homogenized eigenvalues of a periodic composite medium. A convergence proof. Proc. Roy. Soc. Edinburg 127 (1997) 1263-1295.

[26] O. Oleinik, A. Shamaev and G. Yosifian, Mathematical problems in elasticity and homogenization. North Holland, Amsterdam (1992).

[27] E. Sánchez-Palencia, Non homogeneous media and vibration theory. Springer Verlag, Lecture notes in physics 127 (1980).

[28] F. Santosa and W. Symes, A dispersive effective medium for wave propagation in periodic composites. SIAM J. Appl. Math. 51 (1991) 984-1005.

[29] F. Santosa and M. Vogelius, First-order corrections to the homogenized eigenvalues of a periodic composite medium. SIAM J. Appl. Math. 53 (1993) 1636-1668. 